\title{
Rational families of instanton bundles on $\mathbb{P}^{2 n+1}$
}

\author{
L. Costa, N. Hoffmann, R.M. Miró-Roig and A. Schmitt
}

\begin{abstract}
This paper is devoted to the theory of symplectic instanton bundles on an odd dimensional projective space $\mathbb{P}^{2 n+1}$ with $n \geqslant 2$. We study the 't Hooft instanton bundles introduced by Ottaviani and a new family of instanton bundles which generalizes one introduced on $\mathbb{P}^{3}$ independently by Rao and Skiti. The main result is the determination of the birational types of the moduli spaces of 't Hooft and of Rao-Skiti instanton bundles, respectively. Assuming a conjecture of Ottaviani, we show that the moduli space of all symplectic instanton bundles on $\mathbb{P}^{2 n+1}$ with $n \geqslant 2$ is reducible.
\end{abstract}

\section{Introduction}

The notion of an instanton comes from mathematical physics. It denotes a solution of an equation of motion in classical field theory which describes a particle localized both in space and in time. Mathematically, it is a self-dual connection on a principal bundle on a four-dimensional Riemannian manifold. The Penrose-Ward correspondence identifies instantons on the four-dimensional sphere $S^{4}$ with holomorphic instanton bundles on the three-dimensional complex projective space $\mathbb{P}^{3}$ ([Ati79], [AT77]). This construction turned out to be a major motivation for studying vector bundles on complex projective spaces and similar algebraic varieties. In recent preprints, Jardim, Markushevich, Tikhomirov, and Verbitsky managed to settle fundamental open questions on geometric properties of moduli spaces of instanton bundles on $\mathbb{P}^{3}$, namely smoothness, connectedness, and rationality ([JV11], [MT10], [Tik12]).

The Penrose-Ward correspondence exists also on higher odd-dimensional complex projective spaces. In fact, Salamon, Corrigan, Goddard, and Kent introduced a correspondence between selfdual connections on $\operatorname{Sp}_{n}(\mathbb{C})$-bundles on the $n$-dimensional quaternionic projective space $\mathbb{H P}^{n}$ and symplectic instanton bundles on the complex projective space $\mathbb{P}^{2 n+1}, n \geqslant 1$ ([CS88], [CGK85], [Sala84]). For $n \geqslant 2$, the knowledge about moduli spaces of symplectic instanton bundles on $\mathbb{P}^{2 n+1}$ is much less complete, and it is the aim of the present paper to make some progress in this area.

The starting point is the paper by Ottaviani [Ott96]. Ottaviani introduces the notion of symplectic 't Hooft instanton bundles on $\mathbb{P}^{2 n+1}, n \geqslant 2$, and claims without proof that the closure of the locus of symplectic 't Hooft bundles is an irreducible component of the moduli

Received 7 March 2013, accepted in final form 26 November 2013.

2010 Mathematics Subject Classification Primary 14D21; Secondary 14D20, 14J60

Keywords: Instanton bundle, vector bundles on projective space, moduli space, reducibility, rationality

This journal is (C) Foundation Compositio Mathematica 2014. This article is distributed with Open Access under the terms of the Creative Commons Attribution Non-Commercial License, which permits non-commercial reuse, distribution, and reproduction in any medium, provided that the original work is properly cited. For commercial re-use, please contact the Foundation Compositio Mathematica.

The first and third author were partially supported by MTMM2010-15256, and the second and fourth author by SFB 647 "Space-Time-Matter", project C3 "Algebraic Geometry: Deformations, Moduli and Vector Bundles". The final version of this paper was prepared during the last author's visit to the CRM, Bellaterra. A. Schmitt would like to thank that institute for its hospitality and financial support. 


\section{Costa, N. Hoffmann, R.M. Miró-Roig, A. Schmitt}

space of symplectic instanton bundles. So far, we have not been able to prove this claim, but we have checked it for several values of $n$ and the instanton number $k$ (Remark 3.7). In addition, we construct an irreducible moduli space for symplectic 't Hooft bundles. Our first main result is that this moduli space is stably rational or even rational for many values of $n$ and $k$ (Corollary 4.10).

Next, we define the notion of a symplectic Rao-Skiti (RS) instanton bundle on $\mathbb{P}^{2 n+1}, n \geqslant 1$. Symplectic Rao-Skiti instanton bundles generalize bundles studied by Rao [Rao97] and Skiti [Ski97] on $\mathbb{P}^{3}$. To our knowledge, these instanton bundles haven't been investigated for $n \geqslant 2$, so far. We supply irreducible moduli spaces for symplectic Rao-Skiti instanton bundles and prove their rationality (Corollary 5.9). Another important observation is that there are symplectic Rao-Skiti instanton bundles which are not limits of 't Hooft instanton bundles (Example 3.12). Under the assumption of the claim of Ottaviani (which we have verified in some cases), this implies that the moduli space of symplectic instanton bundles is reducible.

Next, we outline the structure of the paper. In Section 2, we fix notation and briefly recall the definition and basic properties of instanton bundles on projective spaces needed later on. In Section 3, we present the definition and main features of the two irreducible families of symplectic instanton bundles on $\mathbb{P}^{2 n+1}$ that we are going to be interested in, namely, symplectic 't Hooft- and symplectic RS-instanton bundles (see Definition 3.3 and 3.9, respectively). In this section we also verify a central claim by Ottaviani concerning the deformation behavior of 't Hooft instanton bundles with the help of a computer (Remark 3.7). Section 4 deals with the construction (Proposition 4.8) and the rationality (Corollary 4.10) of moduli spaces of 't Hooft instanton bundles. Section 5 contains the determination of the birational type of the moduli stacks (Corollary 5.4) and spaces (Corollary 5.9) of RS-instanton bundles.

\section{Notation}

Throughout this paper, we will work over the field $\mathbb{C}$ of complex numbers. Given a vector space $W$, we will denote by $\mathbb{P}(W)$ the projective space of lines in $W$ and set $\mathbb{P}:=\mathbb{P}^{2 n+1}:=\mathbb{P}\left(\mathbb{C}^{2 n+2}\right)$. We will not distinguish between a vector bundle and its locally free sheaf of sections and use the definition of $\mu$-(semi)stability due to Mumford and Takemoto [OSS80]. Given an integer $k \geqslant 1$, let $J=\left(\begin{array}{cc}0 & I \\ -I & 0\end{array}\right)$ denote the standard symplectic form on $\mathbb{C}^{2 n+2 k}$.

\section{Mathematical instanton bundles}

In this section, we recall the definition of (mathematical) instanton bundles and their description in terms of monads.

DeFinition 2.1. Let $k \geqslant 1$ be an integer. An instanton bundle with charge $k$ (for short, a $k$-instanton bundle) is a vector bundle $E$ on $\mathbb{P}$ satisfying the following properties.

(i) $E$ has rank $2 n$.

(ii) The Chern polynomial of $E$ is $c_{t}(E)=1 /\left(1-t^{2}\right)^{k}$.

(iii) $E$ has natural cohomology in the range $-(2 n+1) \leqslant q \leqslant 0$, that is, for any $q$ in that range, there is at most one integer $i=i(q)$ such that $H^{i}(\mathbb{P}, E(q)) \neq 0$.

(iv) $E$ has trivial splitting type, that is, the restriction of $E$ to a general line is trivial.

Remark 2.2. By [AO94], Proposition 2.11, any instanton bundle is simple. Nevertheless, it is an open question whether it is $\mu$-stable.

Definition 2.3. A vector bundle $E$ on $\mathbb{P}$ is called symplectic, if there exists an isomorphism 


\section{RATIONAL FAMILIES OF INSTANTON BUNDLES ON $\mathbb{P}^{2 n+1}$}

$\varphi: E \longrightarrow E^{*}$ such that $\varphi^{*}=-\varphi$. This is equivalent to the existence of a nondegenerate form $\alpha \in H^{0}\left(\mathbb{P}, \bigwedge^{2} E^{*}\right)$.

For $n=1, E$ is a rank 2 vector bundle with $c_{1}(E)=0$. Thus, $\bigwedge^{2} E=\mathscr{O}_{\mathbb{P}}$ and $H^{0}\left(\bigwedge^{2} E\right)$ admits a nowhere vanishing global section. However, when $n \geqslant 2$, the symplectic structure condition does not follow from the definition. In this paper, we will restrict our attention mainly to symplectic instanton bundles. Using the Beilinson spectral sequence [Bei78], we get the following well-known and useful correspondence between symplectic instanton bundles and self-dual monads.

Proposition 2.4. (i) Any symplectic k-instanton bundle $E$ over $\mathbb{P}$ is the cohomology of a symplectic monad

where the matrix

$$
\mathscr{O}_{\mathbb{P}}(-1)^{\oplus k} \stackrel{J A^{t}}{\longrightarrow} \mathscr{O}_{\mathbb{P}}^{2 n+2 k} \stackrel{A}{\longrightarrow} \mathscr{O}_{\mathbb{P}}(1)^{\oplus k}
$$

$$
A \in \operatorname{Mat}_{k \times(2 n+2 k)}\left(H^{0}\left(\mathbb{P}, \mathscr{O}_{\mathbb{P}}(1)\right)\right)
$$

of linear forms has full rank $k$ at every point of $\mathbb{P}$ and satisfies $A J A^{t}=0$.

Conversely, any such matrix $A$ yields a symplectic monad (1), whose cohomology $E$ is a symplectic $k$-instanton bundle if $E$ has trivial splitting type.

(ii) Abbreviate a monad with cohomology bundle $E$ as in (1) by $M_{E}^{\bullet}$. Then, for two such monads $M_{E}^{\bullet}$ and $N_{F}^{\bullet}$, one has

$$
\operatorname{Hom}_{\mathscr{O}_{\mathbb{P}}}(E, F)=\operatorname{Hom}_{\operatorname{Kom}(\mathbb{P})}\left(M_{E}^{\bullet}, N_{F}^{\bullet}\right),
$$

where $\operatorname{Hom}_{\operatorname{Kom}(\mathbb{P})}$ means morphisms in the category of complexes $\operatorname{Kom}(\mathbb{P})$.

Proof. (i) See [OS86], Corollary 1.4 and Lemma 1.5, and [Hof11], Proposition 2.4.

(ii) See [OS83], Proposition 1.3, or [Hof11], Proposition 2.4.

Proposition 2.5. Let $E$ be a $k$-instanton bundle on $\mathbb{P}$ and let $L \subset \mathbb{P}$ be a linear subspace of dimension $r$. Then, $h^{0}\left(E_{\mid L}\right) \leqslant 2 n+k-r$.

Proof. Assume that there is a linear subspace $L \subset \mathbb{P}$ of dimension $r$ such that $h^{0}\left(E_{\mid L}\right) \geqslant$ $2 n+k-r+1$ and consider the monad

$$
\mathscr{O}_{\mathbb{P}}(-1)^{\oplus k} \stackrel{J A^{t}}{\longrightarrow} \mathscr{O}_{\mathbb{P}}^{2 n+2 k} \stackrel{A}{\longrightarrow} \mathscr{O}_{\mathbb{P}}(1)^{\oplus k}
$$

associated with $E$. After changing basis, if necessary, we can assume that $\mathbb{P}$ has homogeneous coordinates $x_{0}, x_{1}, \ldots, x_{2 n+1}, L$ is given by $x_{r+1}=\cdots=x_{2 n+1}=0$, and

$$
A_{\mid L}=\left(A_{1} \mid A_{2}\right) \in \operatorname{Mat}_{k \times(2 n+2 k)}\left(H^{0}\left(\mathbb{P}, \mathscr{O}_{L}(1)\right)\right)
$$

with $A_{2}=(0) \in \operatorname{Mat}_{k \times(2 n+k-r+1)}\left(H^{0}\left(\mathbb{P}, \mathscr{O}_{L}(1)\right)\right)$. By [BV88], Theorem 2.1, the homogeneous ideal $I \subset \mathbb{C}\left[x_{0}, x_{1}, \ldots, x_{r}\right]$ that is generated by the maximal minors of $A_{1} \in \operatorname{Mat}_{k \times(k+r-1)}\left(H^{0}\left(\mathbb{P}, \mathscr{O}_{L}(1)\right)\right)$ has height $\leqslant r$. This implies that there exist closed points $x \in L$ such that $\operatorname{rank}(A(x))<$ $k$, contradicting the fact that $A$ is the matrix which defines the symplectic monad associated with $E$.

Definition 2.6. Given a symplectic instanton bundle $E$ on $\mathbb{P}$ and a linear subspace $L \subset \mathbb{P}$ of dimension $r$, we will say that $L$ is unstable with maximal order of instability if $h^{0}\left(E_{\mid L}\right)=2 n+k-r$.

Remark 2.7. The upper bound given in the above proposition is sharp. Indeed, we will see that it is attained by symplectic RS-instanton bundles (see Definition 3.9). More precisely, for any 


\section{Costa, N. Hoffmann, R.M. Miró-Roig, A. Schmitt}

symplectic RS-instanton bundle $E$ on $\mathbb{P}$, we will prove the existence of an $n$-dimensional linear subspace $L \subset \mathbb{P}$ with maximal order of instability (Proposition 3.11).

\section{Symplectic 't Hooft- and RS-instanton bundles}

This section is devoted to the introduction of the two kinds of instanton bundles that will be the subject of our research, namely, symplectic 't Hooft- and symplectic RS-instanton bundles on $\mathbb{P}$.

Symplectic 't Hooft bundles on $\mathbb{P}^{3}$ were introduced by A. Hirschowitz and M.S. Narasimhan in [HN82]. They constructed them as the bundles associated via Serre's correspondence with disjoint unions of lines and, as a main achievement, proved their unobstructedness. The notion of symplectic 't Hooft bundles on higher odd dimensional projective spaces $\mathbb{P}$ is due to Ottaviani [Ott96], and we start this section recalling it. To this end, we fix integers $n, k \geqslant 1$. Let

$$
a \in \operatorname{Mat}_{k \times(n+k)}(\mathbb{C})
$$

be a matrix of scalars, and let

$$
\begin{aligned}
D:=\operatorname{diag}\left(l_{1}, \ldots, l_{n+k}\right), & l_{1}, \ldots, l_{n+k} \in H^{0}\left(\mathbb{P}, \mathscr{O}_{\mathbb{P}}(1)\right), \\
D^{\prime}:=\operatorname{diag}\left(l_{1}^{\prime}, \ldots, l_{n+k}^{\prime}\right), & l_{1}^{\prime}, \ldots, l_{n+k}^{\prime} \in H^{0}\left(\mathbb{P}, \mathscr{O}_{\mathbb{P}}(1)\right),
\end{aligned}
$$

be diagonal matrices with entries in $H^{0}\left(\mathbb{P}, \mathscr{O}_{\mathbb{P}}(1)\right)$. We consider the matrix

$$
A:=a \cdot\left(D \mid D^{\prime}\right) \in \operatorname{Mat}_{k \times(2 n+2 k)}\left(H^{0}\left(\mathbb{P}, \mathscr{O}_{\mathbb{P}}(1)\right)\right)
$$

as a morphism of vector bundles from $\mathscr{O}_{\mathbb{P}}^{2 n+2 k}$ to $\mathscr{O}_{\mathbb{P}}(1)^{\oplus k}$.

Proposition 3.1 (Ottaviani [Ott96], Section 3). For every choice of $a, l_{j}, l_{j}^{\prime}$, we have

(i) $A J A^{t}=0$;

(ii) the sheaf ker $A(1) \subseteq \mathscr{O}_{\mathbb{P}}(1)^{\oplus(2 n+2 k)}$ has at least $n+k$ global sections.

Moreover, if $a$ and the $l_{j}, l_{j}^{\prime}$ are general, ${ }^{1}$ then we have

(iii) the matrix $A$ has rank $k$ at every point of $\mathbb{P}$;

(iv) if $k \geqslant 3$, then $\operatorname{ker} A(1) \subseteq \mathscr{O}_{\mathbb{P}}(1)^{\oplus(2 n+2 k)}$ has exactly $n+k$ global sections.

Proof. (i) This follows from the calculation

$$
\left(D \mid D^{\prime}\right) \cdot J \cdot\left(D \mid D^{\prime}\right)^{t}=\left(D \mid D^{\prime}\right) \cdot\left(\frac{D^{\prime}}{-D}\right)=D D^{\prime}-D^{\prime} D=0 .
$$

(ii) The previous calculation also implies that $A \cdot J \cdot\left(D \mid D^{\prime}\right)^{t}=0$. Consequently, the $n+k$ columns of the matrix $J \cdot\left(D \mid D^{\prime}\right)^{t}$ are elements in ker $A(1)$. We may assume that the linear forms $l_{j}$ are all nonzero. Then, these columns are linearly independent.

For the rest of the proof, let all $(k \times k)$-minors of $a$ be nonzero, and choose a decomposition

$$
H^{0}\left(\mathbb{P}, \mathscr{O}_{\mathbb{P}}(1)\right)=V \oplus W \quad \text { with } \operatorname{dim} V=\operatorname{dim} W=n+1 .
$$

(iii) Choose $l_{1}, \ldots, l_{n+k} \in V$ such that any $n+1$ of them are a basis of $V$. Consider a point in $\mathbb{P}$ where at least one $v \in V$ does not vanish. Then, at most $n$ of the forms $l_{1}, \ldots, l_{n+k}$ vanish there, so we can find $k$ of them that do not. The corresponding $(k \times k)$-minor of $a \cdot \operatorname{diag}\left(l_{1}, \ldots, l_{n+k}\right)$ is nonzero at this point.

\footnotetext{
${ }^{1}$ By general, we mean that the tuple $\left(a, l_{j}, l_{j}^{\prime}, j=1, \ldots, n+k\right)$ lies in a dense open subset of the affine space $\operatorname{Mat}_{k \times(n+k)}(\mathbb{C}) \oplus H^{0}\left(\mathbb{P}, \mathscr{O}_{\mathbb{P}}(1)\right)^{\oplus 2(n+k)}$.
} 


\section{RATional FAMilies of Instanton BUndLES ON $\mathbb{P}^{2 n+1}$}

Choosing $l_{1}^{\prime}, \ldots, l_{n+k}^{\prime} \in W$ similarly, we can achieve that $a \cdot \operatorname{diag}\left(l_{1}^{\prime}, \ldots, l_{n+k}^{\prime}\right)$ has rank $k$ at all points where at least one $w \in W$ does not vanish. This covers all points in $\mathbb{P}$, thereby proving that statement (iii) holds for some $a$ and $l_{j}, l_{j}^{\prime}$.

(iv) We will again take $l_{1}, \ldots, l_{n+k} \in V$ and $l_{1}^{\prime}, \ldots, l_{n+k}^{\prime} \in W$. A global section

$$
\left(\frac{b \oplus c}{b^{\prime} \oplus c^{\prime}}\right) \in H^{0}\left(\mathbb{P}, \mathscr{O}_{\mathbb{P}}^{2 n+2 k}(1)\right), \quad b, b^{\prime} \in V^{n+k}, \quad c, c^{\prime} \in W^{n+k},
$$

is then in $\operatorname{ker} A(1)$ if and only if it satisfies the system of linear equations

$$
\begin{aligned}
a D b & =0 \in\left(\operatorname{Sym}^{2} V\right)^{k}, \\
a D^{\prime} c^{\prime} & =0 \in\left(\operatorname{Sym}^{2} W\right)^{k}, \\
a\left(D c+D^{\prime} b^{\prime}\right) & =0 \in(V \otimes W)^{k} .
\end{aligned}
$$

Choosing bases $v_{1}, \ldots, v_{n+1} \in V$ and $w_{1}, \ldots, w_{n+1} \in W$, we write

$$
\begin{aligned}
D & =D_{1} v_{1}+\cdots+D_{n+1} v_{n+1}, & \text { with } & D_{1}, \ldots, D_{n+1} \in \operatorname{Mat}_{(n+k) \times(n+k)}(\mathbb{C}), \\
D^{\prime} & =D_{1}^{\prime} w_{1}+\cdots+D_{n+1}^{\prime} w_{n+1}, & \text { with } & D_{1}^{\prime}, \ldots, D_{n+1}^{\prime} \in \operatorname{Mat}_{(n+k) \times(n+k)}(\mathbb{C}) .
\end{aligned}
$$

Specifying the linear forms $l_{1}, \ldots, l_{n+k} \in V$ and $l_{1}^{\prime}, \ldots, l_{n+k}^{\prime} \in W$ is equivalent to specifying the diagonal matrices $D_{1}, \ldots, D_{n+1}$ and $D_{1}^{\prime}, \ldots, D_{n+1}^{\prime}$. We also write

$$
\begin{array}{ll}
b=b_{1} v_{1}+\cdots+b_{n+1} v_{n+1}, & \text { with } \quad b_{i}=\left(b_{i, 1}, \ldots, b_{i, n+k}\right)^{t} \in \mathbb{C}^{n+k}, \\
c=c_{1} w_{1}+\cdots+c_{n+1} w_{n+1}, & \text { with } \quad c_{i}=\left(c_{i, 1}, \ldots, c_{i, n+k}\right)^{t} \in \mathbb{C}^{n+k},
\end{array}
$$

and similarly for $b^{\prime}$ and $c^{\prime}$.

Suppose that $k \geqslant 2$. We claim that (6) has only the trivial solution $b=0$ if $l_{1}, \ldots, l_{n+k} \in V$ are general. It suffices to check this in one special case, say

$$
l_{j}:= \begin{cases}v_{j}, & \text { for } j \leqslant n+1 \\ v_{1}+\cdots+v_{n+1}, & \text { for } j \geqslant n+2 .\end{cases}
$$

This choice translates into

$$
D_{i}=\operatorname{diag}(\underbrace{0, \ldots, 0}_{i-1}, 1, \underbrace{0, \ldots, 0}_{n+1-i}, \underbrace{1, \ldots, 1}_{k-1}) .
$$

The coefficient for $v_{i}^{2}$ in (6) reads $a D_{i} b_{i}=0$. As the relevant $(k \times k)$-minor of $a$ is nonzero, this implies that $D_{i} b_{i}=0$ and, hence, that

$$
b_{i, i}=b_{i, n+2}=b_{i, n+3}=\cdots=b_{i, n+k}=0 .
$$

Given indices $1 \leqslant i_{1}<i_{2} \leqslant n+1$, the coefficient for $v_{i_{1}} v_{i_{2}}$ in (6) reads

$$
a\left(D_{i_{1}} b_{i_{2}}+D_{i_{2}} b_{i_{1}}\right)=0 \in \mathbb{C}^{k} .
$$

Using (9), we see that $D_{i_{1}} b_{i_{2}}+D_{i_{2}} b_{i_{1}} \in \mathbb{C}^{n+k}$ has at most $2 \leqslant k$ nonzero components; as $(k \times k)$-minors of $a$ are nonzero, we can conclude that

$$
D_{i_{1}} b_{i_{2}}+D_{i_{2}} b_{i_{1}}=0 \in \mathbb{C}^{n+k}
$$

and, hence, that $b_{i_{1}, i_{2}}=b_{i_{2}, i_{1}}=0$. This shows that $b=0$ is the only solution of (6) for this particular choice, and, consequently, for a general choice, of $l_{1}, \ldots, l_{n+k} \in V$. Similarly, $c^{\prime}=0$ is the only solution of (7) for a general choice of $l_{1}^{\prime}, \ldots, l_{n+k}^{\prime} \in W$. 


\section{Costa, N. Hoffmann, R.M. Miró-Roig, A. Schmitt}

Suppose that $k \geqslant 3$. It remains to show that (8) has only $n+k$ linearly independent solutions $\left(b^{\prime}, c\right)$, if $l_{1}, \ldots, l_{n+k} \in V$ and $l_{1}^{\prime}, \ldots, l_{n+k}^{\prime} \in W$ are general. Again, it suffices to check this in one special case, say

$$
l_{j}:=\left\{\begin{array}{ll}
v_{j}, & \text { for } j \leqslant n \\
v_{n}, & \text { for } j=n+1 \\
v_{n+1}, & \text { for } j \geqslant n+2
\end{array} \text { and } \quad l_{j}^{\prime}:= \begin{cases}w_{j}, & \text { for } j \leqslant n+1 \\
w_{n}, & \text { for } j=n+2 \\
w_{n+1}, & \text { for } j \geqslant n+3 .\end{cases}\right.
$$

This choice translates into

$$
\begin{aligned}
D_{i}=D_{i}^{\prime} & =\operatorname{diag}(\overbrace{0, \ldots, 0}^{i-1}, 1, \overbrace{0, \ldots, 0,0,0,0,0, \ldots, 0}^{n+k-i}), \quad \text { for } i \leqslant n-1, \\
D_{n} & =\operatorname{diag}(0, \ldots, 0,0,0, \ldots, 0,1,1,0,0, \ldots, 0), \\
D_{n+1} & =\operatorname{diag}(0, \ldots, 0,0,0, \ldots, 0,0,0,1,1, \ldots, 1), \\
D_{n}^{\prime} & =\operatorname{diag}(0, \ldots, 0,0,0, \ldots, 0,1,0,1,0, \ldots, 0), \\
D_{n+1}^{\prime} & =\operatorname{diag}(\underbrace{0, \ldots, 0,0,0, \ldots, 0}_{n-1}, 0,1,0, \underbrace{1, \ldots, 1}_{k-2}) ;
\end{aligned}
$$

note that $D_{1}+\cdots+D_{n+1}$ and $D_{1}^{\prime}+\cdots+D_{n+1}^{\prime}$ both equal the identity matrix.

Given indices $1 \leqslant i_{1}, i_{2} \leqslant n+1$, the coefficient for $v_{i_{1}} \otimes w_{i_{2}}$ in (8) reads

$$
a\left(D_{i_{1}} c_{i_{2}}+D_{i_{2}}^{\prime} b_{i_{1}}^{\prime}\right)=0 \in \mathbb{C}^{k} .
$$

Due to the particular choice we have made, $D_{i_{1}} c_{i_{2}}+D_{i_{2}}^{\prime} b_{i_{1}}^{\prime} \in \mathbb{C}^{n+k}$ has at most $k$ nonzero components; as $(k \times k)$-minors of $a$ are nonzero, we can conclude that

$$
D_{i_{1}} c_{i_{2}}+D_{i_{2}}^{\prime} b_{i_{1}}^{\prime}=0 \in \mathbb{C}^{n+k} .
$$

Taking separately the sum over all $i_{2}$ and the sum over all $i_{1}$, we get

$$
b_{i_{1}}^{\prime}=-D_{i_{1}}\left(c_{1}+\cdots+c_{n+1}\right) \quad \text { and } \quad c_{i_{2}}=-D_{i_{2}}^{\prime}\left(b_{1}^{\prime}+\cdots+b_{n+1}^{\prime}\right) .
$$

Plugging the sum over all $i_{1}$ of the former equation into the latter, we conclude that

$$
c_{i_{2}}=D_{i_{2}}^{\prime}\left(c_{1}+\cdots+c_{n+1}\right) .
$$

This shows that every solution $\left(b^{\prime}, c\right)$ of $(8)$ satisfies

$$
b^{\prime}=-D\left(c_{1}+\cdots+c_{n+1}\right) \text { and } c=D^{\prime}\left(c_{1}+\cdots+c_{n+1}\right) .
$$

As $c_{1}+\cdots+c_{n+1} \in \mathbb{C}^{n+k}$, there are only $n+k$ linearly independent solutions.

Remark 3.2. The hypothesis $k \geqslant 3$ in Proposition 3.1 (iv) cannot be dropped. In fact, it is trivial to check that if $k=1$, then ker $A(1) \subseteq \mathscr{O}_{\mathbb{P}}(1)^{\oplus(2 n+2 k)}$ has exactly $2 n^{2}+3 n+1$ global sections; and it follows from [AO94], Theorem 3.14, that if $k=2$, then $h^{0}(\mathbb{P}, \operatorname{ker} A(1))=2 n+2$.

Definition 3.3 ([Ott96], Section 3). A symplectic $k$-instanton bundle $E$ on $\mathbb{P}$ given by a monad

$$
\mathscr{O}_{\mathbb{P}}(-1)^{\oplus k} \stackrel{J A^{t}}{\longrightarrow} \mathscr{O}_{\mathbb{P}}^{2 n+2 k} \stackrel{A}{\longrightarrow} \mathscr{O}_{\mathbb{P}}(1)^{\oplus k}
$$

is called an 't Hooft instanton bundle if $A$ is of the form (5) introduced above.

Remark 3.4. (i) Proposition 3.1 (i) and (iii) shows that general data

$$
a \in \operatorname{Mat}_{k \times(n+k)}(\mathbb{C}) \text { and } l_{1}, \ldots, l_{n+k}, l_{1}^{\prime}, \ldots, l_{n+k}^{\prime} \in H^{0}\left(\mathbb{P}, \mathscr{O}_{\mathbb{P}}(1)\right)
$$

actually define a symplectic 't Hooft $k$-instanton bundle $E$, via (3), (4), (5) and (1). The condition on the trivial splitting type follows from the other conditions ([Ott96], Corollary 3.5). 


\section{RATional FAMilies of InStANTON BUNDLES ON $\mathbb{P}^{2 n+1}$}

(ii) It follows from [Ott96], Theorem 3.6, that any 't Hooft instanton bundle on $\mathbb{P}$ is stable.

The following statement is also contained in [Ott96], Proposition 3.3 and Theorem 3.7.

Proposition 3.5. Let $E$ be a symplectic 't Hooft $k$-instanton bundle. Then

$$
h^{0}(\mathbb{P}, E(1)) \geqslant n
$$

with equality if $k \geqslant 3$ and $E$ is general.

Proof. Let $E$ be a symplectic $k$-instanton bundle, given as the cohomology of the monad (1). Then, $E(1)$ is the cohomology of a monad

$$
\mathscr{O}_{\mathbb{P}}^{\oplus k} \longrightarrow \mathscr{O}_{\mathbb{P}}(1)^{\oplus(2 n+2 k)} \stackrel{A(1)}{\longrightarrow} \mathscr{O}_{\mathbb{P}}(2)^{\oplus k} .
$$

Using $H^{1}\left(\mathbb{P}, \mathscr{O}_{\mathbb{P}}^{\oplus k}\right)=0$, this implies, in particular, that

$$
h^{0}(\mathbb{P}, E(1))=h^{0}(\mathbb{P}, \operatorname{ker} A(1))-k .
$$

Hence, the claim follows from Proposition 3.1 (ii) and (iv).

Remark 3.6. The hypothesis $k \geqslant 3$ in the above proposition cannot be dropped. In fact, let $E$ be a symplectic $k$-instanton bundle on $\mathbb{P}^{2 n+1}$. By Remark 3.2 , we know that if $k=1(k=2)$, then $h^{0}(\mathbb{P}, \operatorname{ker} A(1))=2 n^{2}+3 n+1\left(h^{0}(\mathbb{P}, \operatorname{ker} A(1))=2 n+2\right)$ and, hence, $h^{0}(\mathbb{P}, E(1))=2 n^{2}+3 n$ $\left(h^{0}(\mathbb{P}, E(1))=2 n\right.$, respectively $)$.

Remark 3.7 (Deformations of 't Hooft instantons). Let $E$ be a general symplectic 't Hooft $k$ instanton bundle over $\mathbb{P}$. Ottaviani claims in [Ott96] that every infinitesimal deformation of $E$ as a symplectic bundle comes from a deformation as a symplectic 't Hooft bundle for $n \geqslant 2$ and $k \geqslant 9$. This would mean that (the closure of) the locus of 't Hooft bundles is an irreducible component of the moduli space of symplectic instanton bundles and would be very interesting in view of our rationality result 4.10. Unfortunately, there is not much of a proof for that in [Ott96], just a reference to a future paper which has not yet appeared.

The claim of Ottaviani in question is equivalent to

$$
\begin{aligned}
& \operatorname{dim}_{\mathbb{C}}\left\{X \in \operatorname{Mat}_{k \times(2 n+2 k)}\left(H^{0}\left(\mathbb{P}, \mathscr{O}_{\mathbb{P}}(1)\right)\right) \mid A \cdot J \cdot X^{t} \text { is symmetric }\right\} \\
& =\left(5 k n+4 n^{2}\right)+\operatorname{dim}\left(\mathrm{GL}_{k}(\mathbb{C}) \times \operatorname{Sp}_{2 n+2 k}(\mathbb{C})\right) \\
& =(n+k) \cdot(6 n+3 k+1),
\end{aligned}
$$

where $A$ is still a matrix of the form (5) above, with general parameters $a$ and $l_{j}, l_{j}^{\prime}$. Using MAPLE, we checked this claim for the following values of $(n, k)$ :

$$
(2,9),(2,10),(3,6),(3,7),(3,8),(3,9),(4,5),(4,6),(4,7),(5,5),(5,6) \text {. }
$$

In these cases, the closure of the locus of 't Hooft bundles is an irreducible component of the moduli space of symplectic instanton bundles. (Note that the reducibility of the moduli space of all instanton bundles was established in [AO00].)

We devote the last part of this section to the introduction of symplectic RS-instanton bundles on $\mathbb{P}$. For $n=1$, symplectic RS-instanton bundles were introduced and studied independently by Rao in [Rao97] and by Skiti in [Ski97]. They are characterized as rank two $k$-instanton bundles on $\mathbb{P}^{3}$ with a jumping line of maximal order, namely, of order $k$. The moduli space of $k$-instantons on $\mathbb{P}^{3}$ has a natural stratification by the maximal order of a jumping line for $E$, and Rao and Skiti proved that the stratum that consists of $k$-instantons having a jumping line of the maximal 


\section{Costa, N. Hoffmann, R.M. Miró-Roig, A. Schmitt}

possible order $k$ is an irreducible rational variety of dimension $6 k+2$. Our next goal will be to introduce the notion of symplectic RS-instanton bundles on $\mathbb{P}$ and study some of their properties.

Again, we fix integers $k, n \geqslant 1$. Let

$$
H:=\left(h_{i j}\right) \in \operatorname{Mat}_{k \times(n+k)}\left(H^{0}\left(\mathbb{P}, \mathscr{O}_{\mathbb{P}}(1)\right)\right)
$$

be a persymmetric matrix of linear forms $h_{i j} \in H^{0}\left(\mathbb{P}, \mathscr{O}_{\mathbb{P}}(1)\right)$, that is, a matrix such that $h_{i j}=h_{s t}$ if $i+j=s+t$. Let

$$
L:=\left\{f_{0}=f_{1}=\cdots=f_{n}=0\right\}
$$

be a linear $n$-space which contains no zeros of the maximal minors of $H$. We consider the matrices

$$
F:=\left(\begin{array}{cccccccc}
f_{0} & f_{1} & \cdots & f_{n} & 0 & \cdots & \cdots & 0 \\
0 & f_{0} & f_{1} & \cdots & f_{n} & 0 & \cdots & 0 \\
0 & 0 & f_{0} & f_{1} & \cdots & f_{n} & \ddots & \vdots \\
\vdots & \cdots & \ddots & \ddots & \ddots & \cdots & \ddots & 0 \\
0 & \cdots & \cdots & 0 & f_{0} & f_{1} & \cdots & f_{n}
\end{array}\right)
$$

and

$$
A:=(F \mid H) \in \operatorname{Mat}_{k \times(2 n+2 k)}\left(H^{0}\left(\mathbb{P}, \mathscr{O}_{\mathbb{P}}(1)\right)\right) .
$$

The matrix $A$ will be considered as a morphism of vector bundles from $\mathscr{O}_{\mathbb{P}}^{2 n+2 k}$ to $\mathscr{O}_{\mathbb{P}}(1)^{\oplus k}$ and it satisfies the following properties.

Proposition 3.8. For general choices of $f_{s} \in H^{0}\left(\mathbb{P}, \mathscr{O}_{\mathbb{P}}(1)\right), 0 \leqslant s \leqslant n$, and of the persymmetric matrix $H$ in (11), we have

(i) $A J A^{t}=0$;

(ii) the sheaf ker $A_{L} \subseteq \mathscr{O}_{L}^{2 n+2 k}$ has exactly $n+k$ global sections;

(iii) the matrix $A$ has rank $k$ at every point $x$ of $\mathbb{P}$.

Proof. (i) This part follows from the calculation

$$
(F \mid H) \cdot J \cdot(F \mid H)^{t}=(F \mid H) \cdot\left(\frac{H^{t}}{-F^{t}}\right)=F H^{t}-H F^{t}=0 .
$$

(ii) This is due to the fact that

$$
A_{L}=\left(0 \mid H_{L}\right)
$$

has exactly $n+k$ independent syzygies of degree 0. (In general, we denote by $\operatorname{Syz}_{d}(A)$ the space of relations $\sum_{i=1}^{q} \alpha_{i} \cdot c_{i}=0$ among the column vectors $c_{1}, \ldots, c_{q}$ of the matrix $A$ with $\operatorname{deg}\left(\alpha_{i}\right)=d$, $i=1, \ldots, q, d \geqslant 0$.)

(iii) We deduce this from the fact that the linear space $L=\left\{f_{0}=f_{1}=\cdots=f_{n}=0\right\}$ contains no zeros of the maximal minors of $H$.

Definition 3.9. A symplectic $k$-instanton bundle $E$ on $\mathbb{P}$ given by a monad

$$
\mathscr{O}_{\mathbb{P}}(-1)^{\oplus k} \stackrel{J A^{t}}{\longrightarrow} \mathscr{O}_{\mathbb{P}}^{2 n+2 k} \stackrel{A}{\longrightarrow} \mathscr{O}_{\mathbb{P}}(1)^{\oplus k}
$$

is called an $R S$-instanton bundle if $A$ is of the form (13) introduced above.

Remark 3.10. (i) Examples of symplectic RS-instanton bundle $E$ on $\mathbb{P}$ include the special symplectic instanton bundles introduced by Spindler and Trautmann in [ST90], Definition 4.1. 


\section{RATional FAMilies of Instanton BUndLES ON $\mathbb{P}^{2 n+1}$}

(ii) Since any symplectic RS-instanton on $\mathbb{P}$ is determined by the choice of general linear forms $f_{s} \in H^{0}\left(\mathbb{P}, \mathscr{O}_{\mathbb{P}}(1)\right), 0 \leqslant s \leqslant n$, and of a general persymmetric matrix $H$ as in (11), we immediately get that the family of symplectic RS-instanton bundles is irreducible. This irreducibility together with the fact that special symplectic instanton bundles are stable [AO94] allows us to conclude that there exists a nonempty open subset of stable symplectic RS-instanton bundles.

In the next proposition we are going to prove that the bound given in Proposition 2.5 is sharp.

Proposition 3.11. Let $E$ be a symplectic $R S$-instanton bundle on $\mathbb{P}$. There exists an unstable $n$-dimensional linear subspace $L \subset \mathbb{P}$ with maximal order of instability. Moreover, if $E$ is general, then $L$ is unique.

Proof. Let $E$ be a symplectic RS-instanton bundle $E$ on $\mathbb{P}$ associated with an $n$-space $L=\left\{f_{0}=\right.$ $\left.f_{1}=\cdots=f_{n}=0\right\}$. By Proposition $2.5, h^{0}\left(\left.E\right|_{L}\right) \leqslant n+k$. On the other hand, it is easy to see, using Proposition 3.8 and the display of the monad associated with $E$ (see [OSS80], page 239), that $h^{0}\left(\left.E\right|_{L}\right)$ equals the number of linearly independent linear syzygies of the matrix $A$ evaluated on $f_{0}=f_{1}=\cdots=f_{n}=0$. Hence, $h^{0}\left(\left.E\right|_{L}\right) \geqslant n+k$ and thus we get the equality.

It remains to prove the uniqueness. Let $E$ be a general symplectic RS-instanton bundle on $\mathbb{P}$. After a change of coordinates, if necessary, we can assume without loss of generality that $E$ is the cohomology of the monad

$$
\mathscr{O}_{\mathbb{P}}(-1)^{\oplus k} \stackrel{J A^{t}}{\longrightarrow} \mathscr{O}_{\mathbb{P}}^{2 n+2 k} \stackrel{A}{\longrightarrow} \mathscr{O}_{\mathbb{P}}(1)^{\oplus k}
$$

where $A=(F \mid H)$ with $H=\left(h_{i j}\right)$ a persymmetric matrix of linear forms and

$$
F=\left(\begin{array}{cccccccc}
x_{0} & x_{1} & \cdots & x_{n} & 0 & \cdots & \cdots & 0 \\
0 & x_{0} & x_{1} & \cdots & x_{n} & 0 & \cdots & 0 \\
0 & 0 & x_{0} & x_{1} & \cdots & x_{n} & \ddots & \vdots \\
\vdots & \cdots & \ddots & \ddots & \ddots & \cdots & \ddots & 0 \\
0 & \cdots & \cdots & 0 & x_{0} & x_{1} & \cdots & x_{n}
\end{array}\right),
$$

where $x_{0}, \ldots, x_{2 n+1}$ are homogeneous coordinates on $\mathbb{P}$. Let $L$ be the $n$-space $\left\{x_{0}=x_{1}=\cdots=\right.$ $\left.x_{n}=0\right\}$. It follows from the first part of the proof that $h^{0}\left(\left.E\right|_{L}\right)=2 n+k-n=n+k$. The fact that $L$ is the unique $n$-space with this property results from the following

Claim. For any $n$-space $L^{\prime} \subset \mathbb{P}, L^{\prime} \neq L, h^{0}\left(\left.E\right|_{L^{\prime}}\right)<n+k$.

We now prove the claim. Since $L^{\prime} \neq L$, there exists an $i \in\{0, \ldots, n\}$ such that $L^{\prime} \nsubseteq\left\{x_{i}=0\right\}$. Without loss of generality, assume that $i=0$. Associated with the monad (14), we have the two short exact sequences

$$
0 \longrightarrow K \longrightarrow \mathscr{O}_{\mathbb{P}}^{2 n+2 k} \stackrel{A}{\longrightarrow} \mathscr{O}_{\mathbb{P}}(1)^{\oplus k} \longrightarrow 0
$$

and

$$
0 \longrightarrow \mathscr{O}_{\mathbb{P}}(-1)^{\oplus k} \longrightarrow K \longrightarrow E \longrightarrow 0,
$$

where $K$ stands for the kernel of $A$. Restricting both sequences to $L^{\prime}$ and taking cohomology, we see that $h^{0}\left(\left.E\right|_{L^{\prime}}\right)=h^{0}\left(\left.K\right|_{L^{\prime}}\right)$. Since $L^{\prime} \nsubseteq\left\{x_{0}=0\right\}$ and the linear forms $h_{i j}$ are general, we have $h^{0}\left(\left.K\right|_{L^{\prime}}\right) \leqslant n+k-1$. This finishes the proof of the claim and the proposition.

By means of the following example, we will see that there are symplectic RS-instanton bundles which are not limits of symplectic 't Hooft instanton bundles. 


\section{Costa, N. Hoffmann, R.M. Miró-Roig, A. Schmitt}

Example 3.12. Fix homogeneous coordinates $x_{0}, \ldots, x_{n}, y_{0}, \ldots, y_{n}$ on $\mathbb{P}$. Given a complex number $\varepsilon$, we consider the persymmetric matrix

$$
H_{\varepsilon}:=\left(\begin{array}{rccccccl}
\varepsilon y_{1} & 0 & \cdots & 0 & y_{0} & \cdots & y_{n-1} & y_{n} \\
0 & \cdots & 0 & y_{0} & \cdots & y_{n-1} & y_{n} & 0 \\
\vdots & . \cdot & . \cdot & \ldots & . & . \cdot & . & . \\
0 & y_{0} & \cdots & y_{n-1} & y_{n} & 0 & \cdots & 0 \\
y_{0} & \cdots & y_{n-1} & y_{n} & 0 & \cdots & 0 & \varepsilon y_{1}
\end{array}\right)
$$

and the $((2 n+2 k) \times k)$-matrix $A_{\varepsilon}=\left(F \mid H_{\varepsilon}\right)$ with

$$
F=\left(\begin{array}{cccccccc}
x_{0} & x_{1} & \cdots & x_{n} & 0 & \cdots & \cdots & 0 \\
0 & x_{0} & x_{1} & \cdots & x_{n} & 0 & \cdots & 0 \\
0 & 0 & x_{0} & x_{1} & \cdots & x_{n} & \ddots & \vdots \\
\vdots & \cdots & \ddots & \ddots & \ddots & \cdots & \ddots & 0 \\
0 & \cdots & \cdots & 0 & x_{0} & x_{1} & \cdots & x_{n}
\end{array}\right) .
$$

The matrix $A_{0}$ has rank $k$ at every point of $\mathbb{P}$ and defines a symplectic $k$-instanton bundle, namely a special symplectic instanton bundle in the sense of [ST90]. Therefore, if $\varepsilon$ is small enough, then $A_{\varepsilon}$ still defines a symplectic $k$-instanton bundle. Assume that $n \geqslant 2$ and $k \geqslant 3$. If $\varepsilon \neq 0$ is small enough, then the RS-instanton bundle $E_{\varepsilon}$ defined by $A_{\varepsilon}$ is not a limit of 't Hooft instanton bundles. Indeed, this will follow from Proposition 3.5 and the following

Proposition 3.13. Assume that $n \geqslant 2$ and $k \geqslant 3$. If $\varepsilon \neq 0$ is sufficiently small, then the $R S$-instanton bundle $E_{\varepsilon}$ defined by the matrix $A_{\varepsilon}$ satisfies $H^{0}\left(\mathbb{P}, E_{\varepsilon}(1)\right)=0$.

Proof. By definition, $E_{\varepsilon}$ is the cohomology of the monad

$$
\mathscr{O}_{\mathbb{P}}(-1)^{\oplus k} \stackrel{J A_{\varepsilon}^{t}}{\longrightarrow} \mathscr{O}_{\mathbb{P}}^{2 n+2 k} \stackrel{A_{\varepsilon}}{\longrightarrow} \mathscr{O}_{\mathbb{P}}(1)^{\oplus k} .
$$

We have the two short exact sequences

$$
\begin{gathered}
0 \longrightarrow K_{\varepsilon} \longrightarrow \mathscr{O}_{\mathbb{P}}^{2 n+2 k} \stackrel{A_{\varepsilon}}{\longrightarrow} \mathscr{O}_{\mathbb{P}}(1)^{\oplus k}, \\
0 \longrightarrow \mathscr{O}_{\mathbb{P}}(-1)^{\oplus k} \longrightarrow K_{\varepsilon} \longrightarrow E_{\varepsilon} \longrightarrow 0,
\end{gathered}
$$

where $K_{\varepsilon}$ denotes the kernel of $A_{\varepsilon}$. Twisting these short exact sequences by $\mathscr{O}_{\mathbb{P}}(1)$ and taking cohomology, we find that $H^{0}\left(\mathbb{P}, K_{\varepsilon}(1)\right)$ coincides with the space

$$
\operatorname{Syz}_{1}\left(A_{\varepsilon}\right)=\operatorname{ker}\left(H^{0}\left(\mathbb{P}, \mathscr{O}_{\mathbb{P}}(1)^{\oplus(2 n+2 k)}\right) \stackrel{A_{\varepsilon}}{\longrightarrow} H^{0}\left(\mathbb{P}, \mathscr{O}_{\mathbb{P}}(2)^{\oplus k}\right)\right)
$$

of all syzygies of $A_{\varepsilon}$ of degree one, and that its dimension $\operatorname{syz}_{1}\left(A_{\varepsilon}\right)$ satisfies

$$
h^{0}\left(\mathbb{P}, E_{\varepsilon}(1)\right)=\operatorname{syz}_{1}\left(A_{\varepsilon}\right)-k .
$$

Let us prove that the following independent vectors span the $\operatorname{space} \operatorname{Syz}_{1}\left(A_{\varepsilon}\right)$ :

$$
\begin{aligned}
& v_{1}=(-\varepsilon y_{1}, \overbrace{0, \ldots, 0}^{k-2},-y_{0}, \ldots,-y_{n}, x_{0}, \ldots, x_{n}, \overbrace{0, \ldots, 0}^{k-1})^{t}, \\
& v_{i}=(\overbrace{0, \ldots, 0}^{k-i},-y_{0}, \ldots,-y_{n}, \overbrace{0, \ldots, 0}^{2 i-2}, x_{0}, \ldots, x_{n}, \overbrace{0, \ldots, 0}^{k-i})^{t}, \quad 1<i<k, \\
& v_{k}=(-y_{0}, \ldots,-y_{n}, \overbrace{0, \ldots, 0}^{k-2},-\varepsilon y_{1}, \overbrace{0, \ldots, 0}^{k-1}, x_{0}, \ldots, x_{n})^{t} .
\end{aligned}
$$




\section{RATional FAMilies of Instanton BUndLES ON $\mathbb{P}^{2 n+1}$}

Since $A_{\varepsilon} \cdot v_{i}=0$, it is clear that $v_{i}$ belongs to $\operatorname{Syz}_{1}\left(A_{\varepsilon}\right)$ for $i=1, \ldots, k$. Let us see that the other inclusion also holds. We consider an arbitrary vector

$$
v=\left(\alpha_{1}, \ldots, \alpha_{n+k}, \beta_{1}, \ldots, \beta_{n+k}\right)^{t} \in \operatorname{Syz}_{1}\left(A_{\varepsilon}\right)
$$

with $\alpha_{q}, \beta_{q} \in H^{0}\left(\mathbb{P}, \mathscr{O}_{\mathbb{P}}(1)\right)$ for $q=1, \ldots, n+k$. Since $A_{\varepsilon} \cdot v=0$, we have

$$
\begin{aligned}
& \alpha_{1} x_{0}+\alpha_{2} x_{1}+\cdots+\alpha_{n+1} x_{n}+\beta_{k} y_{0}+\cdots+\beta_{n+k} y_{n}+\varepsilon \beta_{1} y_{1}=0, \\
& \alpha_{i} x_{0}+\alpha_{1+i} x_{1}+\cdots+\alpha_{n+i} x_{n}+\beta_{k+1-i} y_{0}+\cdots+\beta_{n+k+1-i} y_{n}=0,1<i<k, \\
& \alpha_{k} x_{0}+\alpha_{1+k} x_{1}+\cdots+\alpha_{n+k} x_{n}+\beta_{1} y_{0}+\cdots+\beta_{n+1} y_{n}+\varepsilon \beta_{n+k} y_{1}=0 .
\end{aligned}
$$

Using the basis $x_{0}, \ldots, x_{n}, y_{0}, \ldots, y_{n}$ of $H^{0}\left(\mathbb{P}, \mathscr{O}_{\mathbb{P}}(1)\right)$, we write

$$
\alpha_{q}=\sum_{j=0}^{n}\left(\alpha_{x_{j}}^{q} x_{j}+\alpha_{y_{j}}^{q} y_{j}\right) \quad \text { and } \quad \beta_{q}=\sum_{j=0}^{n}\left(\beta_{x_{j}}^{q} x_{j}+\beta_{y_{j}}^{q} y_{j}\right)
$$

with $\alpha_{x_{j}}^{q}, \alpha_{y_{j}}^{q}, \beta_{x_{j}}^{q}, \beta_{y_{j}}^{q} \in \mathbb{C}$, for $j=0, \ldots, n$ and $q=1, \ldots, n+k$. We first consider the $\alpha_{x_{j}}^{q}$. Comparing coefficients of $x_{j}^{2}$ in (15) shows that

$$
\alpha_{x_{j}}^{j+1}=\alpha_{x_{j}}^{j+2}=\ldots=\alpha_{x_{j}}^{j+k}=0, \quad j=0, \ldots, n .
$$

Comparing coefficients of $x_{a} \cdot x_{b}$ in (15) shows that

$$
\alpha_{x_{b}}^{a+1}+\alpha_{x_{a}}^{b+1}=\alpha_{x_{b}}^{a+2}+\alpha_{x_{a}}^{b+2}=\ldots=\alpha_{x_{b}}^{a+k}+\alpha_{x_{a}}^{b+k}=0, \quad 0 \leqslant a<b \leqslant n .
$$

We know from (16) that (17) also holds for $a=b$. This generalization of (17) implies that

$$
\begin{array}{ll}
\alpha_{x_{j}}^{q}=-\alpha_{x_{q-1}}^{j+1}=\alpha_{x_{j-1}}^{q+1}, & j=0, \ldots, n, \quad q=1, \ldots, n+k, \quad q-j \leqslant 0, \\
\alpha_{x_{j}}^{q}=-\alpha_{x_{q-k}}^{j+k}=\alpha_{x_{j+1}}^{q-1}, & j=0, \ldots, n, \quad q=1, \ldots, n+k, \quad q-j \geqslant k+1 .
\end{array}
$$

Starting from (16), we thus obtain $\alpha_{x_{j}}^{q}=0$ for $q-j \geqslant 1$, by induction on $q-j$, and also $\alpha_{x_{j}}^{q}=0$ for $q-j \leqslant 0$, by descending induction on $q-j$. This proves that

$$
\alpha_{x_{j}}^{q}=0, \quad j=0, \ldots, n, \quad q=1, \ldots, n+k .
$$

We next consider the $\beta_{y_{j}}^{q}$. Comparing coefficients of all products $y_{j}^{2}$ and $y_{a} \cdot y_{b}$ in (15) yields a system of linear equations

$$
M_{\varepsilon} \cdot\left(\beta_{y_{j}}^{q}\right)=0
$$

where $\left(\beta_{y_{j}}^{q}\right)$ is the column vector consisting of the $(n+1)(n+k)$ unknowns $\beta_{y_{j}}^{q}$, and the entries of the coefficient matrix $M_{\varepsilon}$ depend continuously on $\varepsilon$.

For $\varepsilon=0$, the equations (15) are symmetric in the $\alpha_{q}$ and $\beta_{q}$. Therefore, $M_{0}$ is also the coefficient matrix of the system of linear equations (16) and (17). The latter system has only the trivial solution (18), so $M_{0}$ has maximal rank.

Hence, $M_{\varepsilon}$ has maximal rank for all sufficiently small $\varepsilon \in \mathbb{C}$, and, therefore, the system of linear equations (19) has only the trivial solution

$$
\beta_{y_{j}}^{q}=0, \quad j=0, \ldots, n, \quad q=1, \ldots, n+k .
$$

We finally consider the $\alpha_{y_{j}}^{q}$ and the $\beta_{x_{j}}^{q}$. Due to (18) and (20), we have

$$
\alpha_{q}=\sum_{j=0}^{n} \alpha_{y_{j}}^{q} y_{j} \quad \text { and } \quad \beta_{q}=\sum_{j=0}^{n} \beta_{x_{j}}^{q} x_{j}, \quad q=1, \ldots, n+k .
$$




\section{Costa, N. Hoffmann, R.M. Miró-Roig, A. Schmitt}

Comparing coefficients of $x_{a} \cdot y_{b}$ in the $i$-th equation of the system (15) shows that

$$
\begin{array}{ll}
\alpha_{y_{b}}^{i+a}+\beta_{x_{a}}^{k+1-i+b}=0, \quad & a=0, \ldots, n, \quad b=0, \ldots, n, \\
& i=1, \ldots, k, \quad(1,1) \neq(b, i) \neq(1, k) .
\end{array}
$$

Also, comparing coefficients of $x_{a+1} \cdot y_{b}$ in the $(i-1)$-st equation, we deduce that

$$
\begin{array}{ll}
\beta_{x_{a}}^{k+1-i+b}=\beta_{x_{a+1}}^{k+2-i+b}, & a=0, \ldots, n-1, \quad b=0, \ldots, n, \\
& i=2, \ldots, k, \quad(1,2) \neq(b, i) \neq(1, k) .
\end{array}
$$

Since $n \geqslant 2$ and $k \geqslant 3$, all integers $1, \ldots, n+k-1$ occur as $k+1-i+b$ here. Hence, $\beta_{x_{j}}^{q}$ depends only on $q-j$, or, in other words,

$$
\beta_{x_{j}}^{q}=\gamma_{q-j}, \quad j=0, \ldots, n, \quad q=1, \ldots, n+k,
$$

for some numbers $\gamma_{1-n}, \ldots, \gamma_{n+k} \in \mathbb{C}$. Substituting this back into (21) gives

$$
\begin{array}{ll}
\alpha_{y_{j}}^{q}=-\gamma_{k+1+j-q}, \quad & j=0, \ldots, n, \quad q=1, \ldots, n+k, \\
& (1,1) \neq(j, q) \neq(1, n+k) .
\end{array}
$$

Comparing coefficients of $x_{a} \cdot y_{1}$ in the first and last equation in (15) shows that

$$
\begin{array}{cl}
0=\alpha_{y_{1}}^{1+a}+\beta_{x_{a}}^{k+1}+\varepsilon \beta_{x_{a}}^{1}=\alpha_{y_{1}}^{1+a}+\gamma_{k+1-a}+\varepsilon \gamma_{1-a}, & a=0, \ldots, n, \\
0=\alpha_{y_{1}}^{k+a}+\beta_{x_{a}}^{2}+\varepsilon \beta_{x_{a}}^{n+k}=\alpha_{y_{1}}^{k+a}+\gamma_{2-a}+\varepsilon \gamma_{n+k-a}, & a=0, \ldots, n .
\end{array}
$$

If $a>0$, then $\alpha_{y_{1}}^{1+a}=-\gamma_{k+1-a}$ according to (23), and, hence, $\varepsilon \gamma_{1-a}=0$, by (24). Using the assumption $\varepsilon \neq 0$, we thus obtain

$$
\gamma_{1-n}=\gamma_{2-n}=\ldots=\gamma_{0}=0 .
$$

If $a<n$, then $\alpha_{y_{1}}^{k+a}=-\gamma_{2-a}$ according to (23), and, hence, $\varepsilon \gamma_{n+k-a}=0$ by (25). Using the assumption $\varepsilon \neq 0$, we thus obtain

$$
\gamma_{k+1}=\gamma_{k+2}=\ldots=\gamma_{k+n}=0 .
$$

The remaining special cases $a=0$ of (24), and $a=n$ of (25), give

$$
\begin{aligned}
\alpha_{y_{1}}^{1} & =-\gamma_{k+1}-\varepsilon \gamma_{1}=-\varepsilon \gamma_{1}, \\
\alpha_{y_{1}}^{n+k} & =-\gamma_{2-n}-\varepsilon \gamma_{k}=-\varepsilon \gamma_{k} .
\end{aligned}
$$

Combined with the last four displayed equations, (22) and (23) precisely say

$$
v=\gamma_{1} v_{1}+\gamma_{2} v_{2}+\cdots+\gamma_{k} v_{k} .
$$

This shows that the vectors $v_{1}, \ldots, v_{k}$ span the space $\operatorname{Syz}_{1}\left(A_{\varepsilon}\right)$, as claimed.

Remark 3.14. The fact that there are symplectic 't Hooft bundles which are not symplectic RSinstanton bundles follows from Sections 5 and 6 , where we prove that the family of symplectic 't Hooft bundles on $\mathbb{P}$ with charge $k$ is irreducible of dimension $5 k n+4 n^{2}$ and the family of symplectic RS-instanton bundles on $\mathbb{P}$ with charge $k$ is irreducible of dimension $(4 n+2) \cdot k+$ $4 n^{2}+2 n-4$.

\section{The moduli space of 't Hooft instanton bundles and its birational type}

There are a natural parameter space for the data defining a monad for an 't Hooft instanton bundle, an action of a reductive algebraic group on that parameter space, and a dense open 


\section{RATional FAMilies of Instanton BUndLES ON $\mathbb{P}^{2 n+1}$}

subset of the parameter space which is invariant under the group action, such that two points in this dense open subset lie in the same orbit if and only if they define isomorphic 't Hooft instanton bundles (Lemma 4.7). Thus, the stack and the GIT quotient of the parameter space by the group action can be considered as natural moduli spaces for 't Hooft instanton bundles and shall be investigated in this section.

Consider the symplectic vector space

$$
U:=\mathbb{C}^{2}
$$

and the vector space

$$
V:=\mathbb{C}^{k}
$$

We identify the matrix $a$ in (2) with an element

$$
a \in V^{\oplus(n+k)} .
$$

For each $j$, we identify the pair $l_{j}, l_{j}^{\prime} \in H^{0}\left(\mathbb{P}, \mathscr{O}_{\mathbb{P}}(1)\right)$ in (3) and (4) with a linear map

$$
U^{*} \longrightarrow H^{0}\left(\mathbb{P}, \mathscr{O}_{\mathbb{P}}(1)\right), \quad j \in\{1, \ldots, n+k\} .
$$

Choosing a basis of $H^{0}\left(\mathbb{P}, \mathscr{O}_{\mathbb{P}}(1)\right)$ identifies these linear maps with elements $L_{j} \in U^{\oplus(2 n+2)}$, $j=1, \ldots, n+k$. We put $L=\left(L_{j}\right)_{j=1, \ldots, n+k} \in\left(U^{\oplus(2 n+2)}\right)^{\oplus(n+k)}$. For each element in the vector space

$$
\left(U^{\oplus(2 n+2)} \oplus V\right)^{\oplus(n+k)},
$$

we thus obtain a linear map

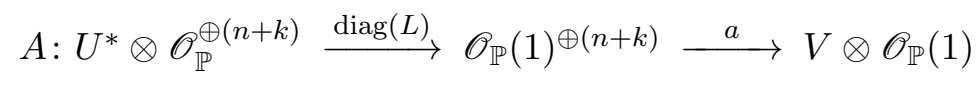

as in (5). It satisfies $A J A^{t}=0$ for the standard symplectic form $J$ on the vector space $\left(U^{*}\right)^{\oplus(n+k)}$.

Consider the linear algebraic group

$$
G_{n, k}:=\left(\mathrm{SL}(U) \times \mathbb{G}_{m}\right) \imath S_{n+k} \times \mathrm{GL}(V) .
$$

Recall that the wreath product $\left(\operatorname{SL}(U) \times \mathbb{G}_{m}\right) \prec S_{n+k}$ is the semidirect product

$$
1 \longrightarrow\left(\mathrm{SL}(U) \times \mathbb{G}_{m}\right)^{\times(n+k)} \longrightarrow\left(\mathrm{SL}(U) \times \mathbb{G}_{m}\right) \imath S_{n+k} \longmapsto S_{n+k} \longrightarrow 1
$$

where $S_{n+k}$ acts on $\left(\operatorname{SL}(U) \times \mathbb{G}_{m}\right)^{\times(n+k)}$ by permuting the factors.

We can write each element $g \in G_{n, k}$ in the form

$$
g=\left(\sigma \cdot\left(\beta_{j}, \gamma_{j}\right)_{j}, \alpha\right)
$$

with $\alpha \in \mathrm{GL}(V), \beta_{j} \in \mathrm{SL}(U), \gamma_{j} \in \mathbb{G}_{m}$ and $\sigma \in S_{n+k}$. It induces an isomorphism

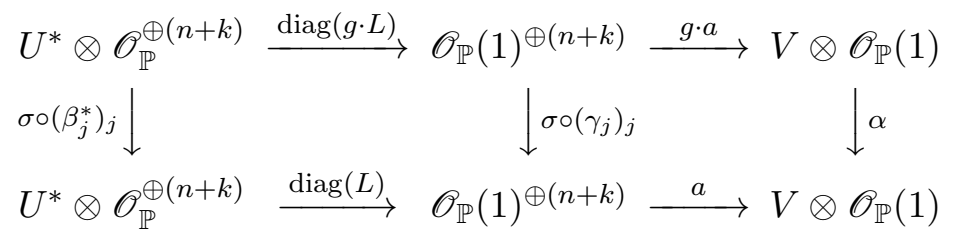

with

$$
\begin{aligned}
(g \cdot a)_{j} & :=\alpha^{-1}\left(\gamma_{j} a_{\sigma(j)}\right), \\
(g \cdot L)_{j} & :=\gamma_{j}^{-1} \beta_{j}\left(L_{\sigma(j)}\right)
\end{aligned}
$$




\section{Costa, N. Hoffmann, R.M. Miró-Roig, A. Schmitt}

These formulas define a linear action of $G_{n, k}$ on $\left(U^{\oplus(2 n+2)} \oplus V\right)^{\oplus(n+k)}$. The subgroup

$$
\mu_{2} \hookrightarrow G_{n, k}
$$

given by the diagonal embedding $\mu_{2} \hookrightarrow\left(\mathrm{SL}(U) \times \mathbb{G}_{m}\right)^{\times(n+k)} \times \mathrm{GL}(V)$ acts trivially.

Proposition 3.1 (iii), states that $R_{n, k} \neq \varnothing$, for the open locus

$$
R_{n, k} \subseteq\left(U^{\oplus(2 n+2)} \oplus V\right)^{\oplus(n+k)}
$$

where $A$ has rank $k$ at every point in $\mathbb{P}$. The subset $R_{n, k}$ is preserved by $G_{n, k}$. Each point in $R_{n, k}$ defines a symplectic monad (1) whose cohomology is a symplectic $k$-instanton bundle $E$; it is constant up to isomorphy on the $G_{n, k}$-orbits. Note that $E$ has trivial splitting type and is stable, by [Ott96], Corollary 3.5 and Theorem 3.6.

Definition 4.1. A stable 't Hooft datum is a point of $R_{n, k}$.

We leave it to the reader to formulate the moduli problem of stable 't Hooft data; compare for example [Hof11], Remark 3.5. In the following, we will demonstrate that the geometric quotient

$$
R_{n, k} / G_{n, k}
$$

exists as a smooth quasi-projective variety. For this, we recall some details of the construction of the moduli space $\mathrm{MI}_{\mathbb{P}^{2 n+1}}(k)$ of stable symplectic $k$-instanton bundles on $\mathbb{P}^{2 n+1}$ contained in [OS86].

Theorem 4.2 (The first fundamental theorem of invariant theory for symplectic groups). Let $E$ and $F$ be finite dimensional complex vector spaces, let $\varphi: F \longrightarrow F^{*}$ be a symplectic form on $F$, and let $S$ be the isometry group of $(F, \varphi)$. Then, the $S$-invariant map

$$
\begin{aligned}
\kappa: \operatorname{Hom}(E, F) & \longrightarrow \operatorname{Hom}_{\mathrm{AS}}\left(E, E^{*}\right) \\
f & \longmapsto f^{*} \circ \varphi \circ f
\end{aligned}
$$

induces a closed embedding of the categorical quotient of the vector space $\operatorname{Hom}(E, F)$ by the action of $S$ into $\operatorname{Hom}_{\mathrm{AS}}\left(E, E^{*}\right)$, the linear subspace of antisymmetric homomorphisms. The image consists of those homomorphisms whose rank is less than or equal to $\min \{\operatorname{dim}(E), \operatorname{dim}(F)\}$.

Proof. [GW09], Theorem 5.2.2 and Lemma 5.2.4.

In the study of monads (1), we look at matrices in the vector space

$$
\mathrm{M}:=\operatorname{Hom}\left(U^{\oplus(n+k)}, V^{\oplus(2 n+2)}\right)
$$

and in the study of symplectic monads, at matrices in the closed subvariety

$$
\mathrm{SM}:=\left\{A \in \mathrm{M} \mid A J A^{t}=0\right\} .
$$

According to Theorem 4.2,

$$
\begin{aligned}
\kappa: \mathbb{P}(\mathrm{M}) & \longrightarrow \mathbb{P}\left(\operatorname{Hom}_{\mathrm{AS}}\left(\left(V^{\oplus(2 n+2)}\right)^{*}, V^{\oplus(2 n+2)}\right)\right) \\
& {[A] \longmapsto\left[K(A):\left(V^{\oplus(2 n+2)}\right)^{*} \stackrel{A^{*}}{\longrightarrow}\left(U^{\oplus(n+k)}\right)^{*} \stackrel{J}{\longrightarrow} U^{\oplus(n+k)} \stackrel{A}{\longrightarrow} V^{\oplus(2 n+2)}\right] }
\end{aligned}
$$

is a model for the categorical quotient of $\mathbb{P}(\mathrm{M})$ by the given $\mathrm{Sp}_{2(n+k)}(\mathbb{C})$-action, possibly followed by a closed embedding.

Remark 4.3. One calls $K(A)$ the Kronecker module of $A$. We may view $K(A)$ as an element of

$$
\mathrm{H}:=\operatorname{Hom}\left(\bigwedge^{2}\left(\mathbb{C}^{2 n+2}\right), \operatorname{Hom}\left(V^{*}, V\right)\right) .
$$




\section{RATional FAMilies of Instanton BUndLES ON $\mathbb{P}^{2 n+1}$}

If $A \in \mathrm{SM}$, then $K(A)$ takes values in the linear subspace $\operatorname{Hom}_{\mathrm{S}}\left(V^{*}, V\right)$ of symmetric homomorphisms, that is, $K(A)$ is a symmetric Kronecker module (compare [OS86], p. 39).

Finally, one has to study the $\mathrm{SL}(V)$-action on $\mathbb{P}(\mathrm{H})$. By work of Hulek [Hul80], a point in $\mathbb{P}(\mathrm{H})$ which comes from a monad whose cohomology is a stable instanton bundle is $\mathrm{SL}(V)$-stable ([OS86], Lemmas 1.11 and 1.12). Let

$$
\mathrm{SI}_{k} \subset \mathbb{P}(\mathrm{H})
$$

be the $\mathrm{SL}(V)$-invariant locally closed subset of points coming from monads with stable $k$-instanton bundles as cohomology. By the aforementioned result of Hulek, the geometric quotient

$$
\operatorname{MI}_{\mathbb{P}^{2 n+1}}(k):=\mathrm{SI}_{k} / \mathrm{SL}(V)
$$

exists as quasi-projective variety. It is the moduli space of stable $k$-instanton bundles on $\mathbb{P}^{2 n+1}$.

Remark 4.4. Set

$$
\widetilde{\mathrm{SI}}_{k}:=\mathbb{P}(\mathrm{SM}) \cap \kappa^{-1}\left(\mathrm{SI}_{k}\right)
$$

and let $\mathscr{M}_{\mathbb{P}^{2 n+1}}(k)$ be the moduli space of stable vector bundles with Chern character $1 /\left(1-t^{2}\right)^{k}$ on $\mathbb{P}=\mathbb{P}^{2 n+1}$. We have the induced morphism

$$
\widetilde{\mathrm{SI}}_{k} \longrightarrow \mathscr{M}_{\mathbb{P}^{2 n+1}}(k) \text {. }
$$

Since this morphism factorizes over the categorical quotient of $\widetilde{\mathrm{SI}}_{k}$ by the action of $\mathrm{Sp}_{2(n+k)}(\mathbb{C}) \times$ PGL $(V)$, Proposition 2.4 (ii), shows that this quotient is actually a geometric one. Remark 2.2 implies further that the quotient map (compare [OST99], Proposition 1.3.1, or [Sch08], Theorem 1.5.3.1, i)

$$
\widetilde{\mathrm{SI}}_{k} \longrightarrow \widetilde{\mathrm{SI}}_{k} /\left(\operatorname{Sp}_{2(n+k)}(\mathbb{C}) \times \operatorname{PGL}(V)\right)=\widetilde{\mathrm{SI}}_{k} /\left(\operatorname{Sp}_{2(n+k)}(\mathbb{C}) \times \mathrm{SL}(V)\right)=\mathrm{SI}_{k} / \operatorname{SL}(V)
$$

is a principal $\left(\mathrm{Sp}_{2(n+k)}(\mathbb{C}) \times \operatorname{PGL}(V)\right)$-bundle.

We now look at the linear $G_{n, k}$-action on

$$
W_{n, k}:=\left(U^{\oplus(2 n+2)} \oplus V\right)^{\oplus(n+k)} .
$$

The subgroup

is normal, and we set

$$
\mathbb{G}_{m}^{\times(n+k+1)} \cong \mathbb{G}_{m}^{n+k} \times \mathscr{Z}(\mathrm{GL}(V)) \subset G_{n, k}
$$

Let us first look at the $\mathbb{G}_{m}^{\times(n+k+1)}$-action on $W_{n, k}$. We use the isogeny

$$
\begin{aligned}
\mathbb{G}_{m}^{\times(n+k)} \times \mathbb{G}_{m} & \longrightarrow \mathbb{G}_{m}^{\times(n+k)} \times \mathbb{G}_{m} \\
\left(\left(\gamma_{j}\right)_{j=1, \ldots, n+k}, z\right) & \longmapsto\left(\left(z^{-1} \cdot \gamma_{j}\right)_{j=1, \ldots, n+k}, z^{2}\right) .
\end{aligned}
$$

Then, the last factor just acts by multiplying everything by $z, z \in \mathbb{G}_{m}$, and the $\mathbb{G}_{m}^{\times(n+k+1)}$ semistable points in $W_{n, k}$ correspond to the $\mathbb{G}_{m}^{\times(n+k)}$-semistable points in $\mathbb{P}\left(W_{n, k}\right)$.

Lemma 4.5. For a point $p=\left[\left(L_{j}\right)_{j=1, \ldots, n+k}, a\right] \in \mathbb{P}\left(W_{n, k}\right)$, the following conditions are equivalent.

(i) The point $p$ is semistable with respect to the action of $\mathbb{G}_{m}^{\times(n+k)}$.

(ii) The point $p$ is stable with respect to the action of $\mathbb{G}_{m}^{\times(n+k)}$.

(iii) $\forall j \in\{1, \ldots, n+k\}: a_{j} \neq 0$ and $L_{j} \neq 0$. 


\section{Costa, N. Hoffmann, R.M. Miró-Roig, A. Schmitt}

Proof. By (26) and (27), the $j$-th copy of $\mathbb{G}_{m}$ in $\mathbb{G}_{m}^{\times(n+k)}$ multiplies the $j$-th column of $a \in$ $V^{\oplus(n+k)}$ by $z$ and $L_{j}$ by $z^{-1}, z \in \mathbb{G}_{m}, j=1, \ldots, n+k$. With this information about the group action, the proof is straightforward.

As before, we may view a tuple $\left(L_{j}\right)_{j=1, \ldots, n+k} \in\left(U^{\oplus(2 n+2)}\right)^{\oplus(n+k)}$ as a matrix $\left(D \mid D^{\prime}\right)$ where $D$ and $D^{\prime}$ are diagonal matrices of the format $(n+k) \times(n+k)$ with entries in $H^{0}\left(\mathbb{P}, \mathscr{O}_{\mathbb{P}}(1)\right)$. We define

$$
\begin{aligned}
\alpha: W_{n, k} & \longrightarrow \mathrm{M} \\
\left(\left(L_{j}\right)_{j=1, \ldots, n+k}, a\right) & \longmapsto a \cdot\left(D \mid D^{\prime}\right) .
\end{aligned}
$$

The map $\alpha$ is invariant under the $\mathbb{G}_{m}^{\times(n+k)}$-action, and the semistability condition in Lemma 4.5 clearly implies that $\alpha(p) \neq 0$ holds for every $\mathbb{G}_{m}^{\times(n+k)}$-semistable point $p=\left(\left(L_{j}\right)_{j=1, \ldots, n+k}, a\right) \in$ $W_{n, k}$. Therefore, $\alpha$ descends to a morphism

$$
\bar{\alpha}: \bar{W}_{n, k}:=W_{n, k} / \mathbb{G}_{m}^{\times(n+k+1)} \longrightarrow \mathbb{P}(\mathrm{M})
$$

between projective varieties, and the image of $\bar{\alpha}$ is contained in the closed subvariety $\mathbb{P}(\mathrm{SM})$. Using Lemma 4.5, one checks that $\bar{\alpha}$ is injective, too.

The group $H_{n, k}$ acts on the quotient $\bar{W}_{n, k}$. There is a natural linearization of this action. Note that the vector space $U$ is equipped with the symplectic form $U \times U \longrightarrow \mathbb{C},\left(u, u^{\prime}\right) \longmapsto$ $\operatorname{det}\left(u \mid u^{\prime}\right)$. Our convention is also such that the symplectic form on $U^{\oplus(n+k)}$ is the direct sum of the symplectic forms on the summands. In this way, $H_{n, k}$ becomes a subgroup of $\operatorname{Sp}_{2(n+k)}(\mathbb{C}) \times$ $\operatorname{PGL}(V)$.

Remark 4.6. It is straightforward to compute the GIT-semistable and stable points for the $H_{n, k}$-action on $\bar{W}_{n, k}$. Unfortunately, the condition one finds is weaker than the condition of $\left(\mathrm{Sp}_{2(n+k)}(\mathbb{C}) \times \mathrm{PGL}(V)\right)$-semistability and stability on $\mathbb{P}(\mathrm{M})$. For example, using the first fundamental theorem of invariant theory (Theorem 4.2), we can determine the $\operatorname{SL}(U)^{\times(n+k)}$ - and $\mathrm{Sp}_{2(n+k)}(\mathbb{C})$-semistable points. The $\mathrm{Sp}_{2(n+k)}(\mathbb{C})$-semistable points in $\mathbb{P}(\mathrm{M})$ are just those points

$$
[A] \in \mathbb{P}(\mathrm{M}), \quad A: U^{\oplus(n+k)} \longrightarrow V^{\oplus(2 n+2)},
$$

for which the image of $A^{*}$ is not an isotropic subspace of $\left(U^{\oplus(n+k)}\right)^{*}$. Viewing $A$ as a tuple of linear maps

$$
A_{i}: U \longrightarrow V^{\oplus(2 n+2)}, \quad i=1, \ldots, n+k,
$$

we see that $[A]$ is $\operatorname{SL}(U)^{\times(n+k)}$-semistable, if and only if the image of $A_{i}^{*}$ is not an isotropic subspace of $U$, that is, $A_{i}^{*}$ is surjective, $i=1, \ldots, n+k$. It is now clear that the $\operatorname{SL}(U)^{\times(n+k)_{-}}$ semistability of $[A]$ will in general not imply its $\operatorname{Sp}_{2(n+k)}(\mathbb{C})$-semistability, not even if $[A]$ lies in the image of $\bar{\alpha}$.

Unfortunately, this means that the GIT-quotient $\bar{W}_{n, k} / / H_{n, k}$ will not map to the GITquotient $\mathbb{P}(\mathrm{M}) / /\left(\mathrm{Sp}_{2(n+k)}(\mathbb{C}) \times \mathrm{PGL}(V)\right)$. This makes it harder to understand the map from the moduli space of stable 't Hooft data to the moduli space of stable symplectic instanton bundles (compare Proposition 4.8).

One should compare this situation with the situation of instantons in general. On the one hand, monads can be considered as quiver representations, and King's results [Kin94] give a parameter dependent notion of stability for these monads. The results in [CO03] show that the monad of an instanton bundle is stable with respect to all parameters. On the other hand, it is still unknown, if an instanton bundle is stable as a vector bundle. 


\section{RATional FAMilies of InStANTON BUNDLES ON $\mathbb{P}^{2 n+1}$}

On the positive side, the facts that $\bar{\alpha}$ is finite and equivariant and that $H_{n, k}$ is a subgroup of $\operatorname{Sp}_{2(n+k)}(\mathbb{C}) \times \operatorname{PGL}(V)$ imply that a point $p \in W_{n, k} / \mathbb{G}_{m}^{\times(n+k+1)}$ whose image $\bar{\alpha}(p) \in \mathbb{P}(M)$ is $\operatorname{Sp}_{2(n+k)}(\mathbb{C}) \times \mathrm{PGL}(V)$-stable is $H_{n, k}$-stable. This applies, in particular, to the points of the open subset

$$
\bar{R}_{n, k}:=R_{n, k} / \mathbb{G}_{m}^{\times(n+k+1)} \subset W_{n, k} / \mathbb{G}_{m}^{\times(n+k+1)}=\bar{W}_{n, k}
$$

(compare Remark 4.4). Therefore, the geometric quotient

$$
\bar{R}_{n, k} / H_{n, k}
$$

exists as a quasi-projective variety. Remark 4.4 also implies that the quotient map

$$
\bar{R}_{n, k} \longrightarrow \bar{R}_{n, k} / H_{n, k}
$$

is a principal $H_{n, k}$-bundle. Since $R_{n, k} \longrightarrow \bar{R}_{n, k}$ is a $\mathbb{G}_{m}^{\times(n+k+1)}$-bundle, the variety $\bar{R}_{n, k} / H_{n, k}$ is actually smooth. As in [OST99], Proposition 1.3.1, or [Sch08], Theorem 1.5.3.1, i), one checks

$$
\bar{R}_{n, k} / H_{n, k} \cong \mathbb{P}\left(W_{n, k}\right) /\left(G_{n, k} / \mathbb{G}_{m}\right) \cong W_{n, k} / G_{n, k}
$$

In the study of the birational geometry of this quotient, we will need the following open subset. Let

$$
R_{n, k}^{0} \subseteq R_{n, k} \subseteq\left(U^{\oplus(2 n+2)} \oplus V\right)^{\oplus(n+k)}
$$

be the open locus where, moreover, $l_{j}$ and $l_{j}^{\prime}$ are linearly independent for each $j$, the $n+k$ linear subspaces $\left\{l_{j}=l_{j}^{\prime}=0\right\} \subseteq \mathbb{P}$ are distinct, and

$$
\operatorname{ker} A(1) \subseteq U^{*} \otimes \mathscr{O}_{\mathbb{P}}(1)^{\oplus(n+k)}
$$

has exactly $n+k$ global sections. If $k \geqslant 3$, then $R_{n, k}^{0} \neq \varnothing$ due to Proposition 3.1 (iv).

Lemma 4.7. Given two points

$$
(a, L),(\tilde{a}, \tilde{L}) \in R_{n, k}^{0},
$$

with corresponding symplectic instanton bundles $E, \tilde{E}$, every isomorphism

$$
\varphi: \tilde{E} \longrightarrow E
$$

is induced by a unique group element $g \in G_{n, k}$ with $g \cdot(a, L)=(\tilde{a}, \tilde{L})$.

Proof. The isomorphism $\varphi$ comes from a unique isomorphism of symplectic monads

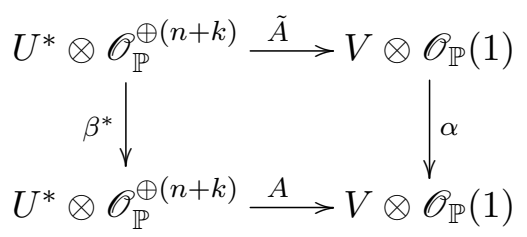

with $\alpha \in \operatorname{GL}(V)$ and $\beta \in \operatorname{Sp}\left(U^{\oplus(n+k)}\right)$; here $A:=a \circ \operatorname{diag}(L)$ and $\tilde{A}:=\tilde{a} \circ \operatorname{diag}(\tilde{L})$.

As we have seen in the proof of Proposition 3.1 (i) and (ii), the $n+k$ global sections of ker $A(1)$ and of ker $\tilde{A}(1)$ are given by the two maps

$$
J \circ \operatorname{diag}(L)^{t} \text { and } J \circ \operatorname{diag}(\tilde{L})^{t}: \mathscr{O}_{\mathbb{P}}^{\oplus(n+k)} \longrightarrow U^{*} \otimes \mathscr{O}_{\mathbb{P}}(1)^{\oplus(n+k)} .
$$




\section{Costa, N. Hoffmann, R.M. Miró-Roig, A. Schmitt}

Since our isomorphism of symplectic monads has to respect these, it has the form

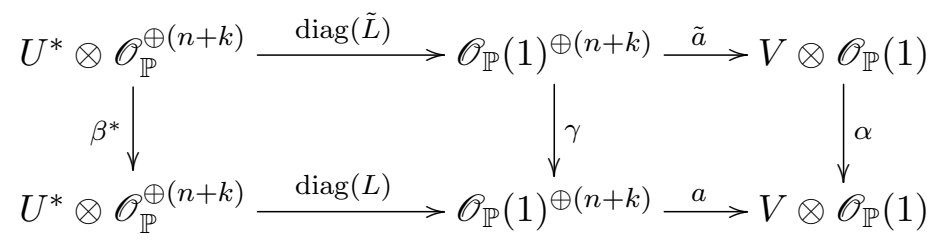

with $\gamma \in \mathrm{GL}_{n+k}(\mathbb{C})$. The loci in $\mathbb{P}$ where $\operatorname{diag}(L)$ and $\operatorname{diag}(\tilde{L})$ are not surjective are

$$
\bigcup_{j}\left\{l_{j}=l_{j}^{\prime}=0\right\} \quad \text { and } \bigcup_{j}\left\{\tilde{l}_{j}=\tilde{l}_{j}^{\prime}=0\right\} .
$$

Since our isomorphisms $\beta$ and $\gamma$ have to respect these, they are of the form

$$
\beta=\sigma \circ\left(\beta_{j}\right)_{j} \text { and } \gamma=\sigma \circ\left(\gamma_{j}\right)_{j}
$$

with $\beta_{j} \in \mathrm{GL}(U), \gamma_{j} \in \mathbb{G}_{m}$, and $\sigma \in S_{n+k}$. Here, $\beta_{j} \in \mathrm{SL}(U)$ as $\beta$ is symplectic.

We summarize our discussion.

Proposition 4.8. Fix positive integers $n$ and $k$. Then, the moduli space

$$
\mathrm{HI}_{\mathbb{P}^{2 n+1}}(k):=R_{n, k} / G_{n, k}
$$

of stable 't Hooft data exists as a smooth quasi-projective scheme and comes with a generically injective morphism $\iota$ to the moduli space $\mathrm{MI}_{\mathbb{P}^{2 n+1}}(k)$ of stable symplectic $k$-instanton bundles on $\mathbb{P}^{2 n+1}$. The moduli space $\mathrm{HI}_{\mathbb{P}^{2 n+1}}(k)$ contains

$$
\mathrm{HI}_{\mathbb{P}^{2 n+1}}^{0}(k):=R_{n, k}^{0} / G_{n, k}
$$

as a dense open subscheme. The morphism $\iota$ is injective on $\mathrm{HI}_{\mathbb{P} 2 n+1}^{0}(k)$.

We are now going to investigate the birational geometry of the moduli space $\mathrm{HI}_{\mathbb{P}^{2 n+1}}(k)$. A quotient of a vector space modulo a group that acts generically freely is the quotient of an open subvariety where the action is free; this is well defined up to birational equivalence.

Theorem 4.9. Assume that $k \geqslant 3$. The coarse moduli scheme $R_{n, k}^{0} / G_{n, k}$ of 'generic' 't Hooft data on $\mathbb{P}^{2 n+1}$ is birational to

$$
\mathbb{C}^{5 k n+4 n^{2}-2^{e}\left(2^{e}+3\right) / 2} \times\left(\frac{\mathrm{Mat}_{2^{e} \times 2^{e}}^{\mathrm{sym}}(\mathbb{C})^{2}}{\mathrm{PO}_{2^{e}}}\right),
$$

where $2^{e}$ is the largest power of 2 that divides both $n$ and $k$.

Here, the projective orthogonal group $\mathrm{PO}_{2^{e}}:=\mathrm{O}_{2^{e}} / \mu_{2}$ acts on the vector space $\mathrm{Mat}_{2^{e} \times 2^{e}}^{\mathrm{sym}}(\mathbb{C})^{2}$ of pairs of symmetric matrices by simultaneous conjugation.

Corollary 4.10. Assume that $k \geqslant 3$ and let $R_{n, k}^{0} / G_{n, k}$ be the coarse moduli scheme of 'generic' 't Hooft data on $\mathbb{P}^{2 n+1}$. The following holds.

(i) If $\operatorname{gcd}(n, k) \not \equiv 0 \bmod 4$, then $R_{n, k}^{0} / G_{n, k}$ is rational.

(ii) If $\operatorname{gcd}(n, k) \not \equiv 0 \bmod 16$, then $R_{n, k}^{0} / G_{n, k}$ is stably rational.

Proof. Saltman [Salt02] proved that the quotient $\mathrm{Mat}_{2^{e} \times 2^{e}}^{\mathrm{sym}}(\mathbb{C})^{2} / \mathrm{PO}_{2^{e}}$ is rational for $2^{e}=1,2$, and stably rational for $2^{e}=4$, and Beneish [Ben05] proved that it is stably rational for $2^{e}=8$. This and Theorem 4.9 imply the corollary. 


\section{RATional FAMilies of InStANTON BUNDLES ON $\mathbb{P}^{2 n+1}$}

Proof of Theorem 4.9. By Lemma 4.7 and Proposition 4.8, we have

$$
\frac{R_{n, k}^{0}}{G_{n, k}} \simeq \frac{\left(U^{\oplus(2 n+2)} \oplus V\right)^{\oplus(n+k)}}{G_{n, k} / \mu_{2}},
$$

where $G_{n, k}=\left(\operatorname{SL}(U) \times \mathbb{G}_{m}\right) \imath S_{n+k} \times \mathrm{GL}(V)$ acts via the formulas (26) and (27).

Let $u_{1}, u_{2}$ be a basis of $U$, and put $u=\left(u_{1}, u_{2}\right) \in U^{2}$. Then, the $G_{n, k}$-orbit of $(u, \ldots, u) \in$ $\left(U^{2}\right)^{n+k}$ is open, with stabilizer $\left(\mu_{2} \imath S_{n+k}\right) \times \mathrm{GL}(V) \subseteq G_{n, k}$. Hence,

$$
\frac{R_{n, k}^{0}}{G_{n, k}} \simeq \frac{\left(U^{2 n} \oplus V\right)^{n+k}}{\left(\left(\mu_{2}\left(S_{n+k}\right) \times \mathrm{GL}(V)\right) / \mu_{2}\right.},
$$

where each copy of $\mu_{2}$ acts trivially on $U^{2 n}$ and via its nontrivial character on $V$.

Here, the group action is already generically free on the direct summand $V^{n+k}$, because the finite group $\left(\mu_{2}<S_{n+k}\right) / \mu_{2}$ acts effectively, and, hence, generically freely, on $V^{n+k} / \mathrm{GL}(V) \simeq$ $\mathrm{Gr}_{k}\left(\mathbb{C}^{n+k}\right)$. Thus, the no-name lemma ([BK86], Lemma 1.2) yields

$$
\begin{aligned}
\frac{R_{n, k}^{0}}{G_{n, k}} & \simeq \frac{V^{n+k}}{\left(\left(\mu_{2} 2 S_{n+k}\right) \times \mathrm{GL}(V)\right) / \mu_{2}} \times \mathbb{C}^{4 n(n+k)} \\
& \simeq \frac{(\mathbb{C} \oplus V)^{n+k}}{\left(\left(\mu_{2} 2 S_{n+k}\right) \times \mathrm{GL}(V)\right) / \mu_{2}} \times \mathbb{C}^{(4 n-1)(n+k)}
\end{aligned}
$$

where each $\mu_{2}$ acts trivially on the corresponding summand $\mathbb{C}$.

Sending $\lambda_{1}, \ldots, \lambda_{n+k} \in \mathbb{C}$ to the matrix $\operatorname{diag}\left(\lambda_{1}, \ldots, \lambda_{n+k}\right)$ defines a morphism

$$
\mathbb{C}^{n+k} \longrightarrow \mathrm{Mat}_{(n+k) \times(n+k)}^{\mathrm{sym}}(\mathbb{C}) .
$$

This morphism is equivariant for the above (permutation) action of $\mu_{2} \prec S_{n+k}$ on $\mathbb{C}^{n+k}$ and its action as a subgroup of $\mathrm{O}_{n+k}$ on $\mathrm{Mat}_{(n+k) \times(n+k)}^{\mathrm{sym}}(\mathbb{C})$ by conjugation.

Given $v_{1}, \ldots, v_{n+k} \in V$, they define a linear map $V^{*} \longrightarrow \mathbb{C}^{n+k}$; sending them to the image of this map whenever it is injective defines a dominant rational map

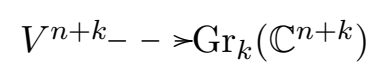

which is invariant under GL( $V)$ and equivariant under $\mu_{2} 2 S_{n+k}$ (this time acting as a subgroup of $\mathrm{O}_{n+k}$, so each $\mu_{2}$ acts nontrivially on one copy of $\mathbb{C}$ ). Taking the product of this morphism and this rational map, we thus obtain a rational map

$$
\frac{(\mathbb{C} \oplus V)^{n+k}}{\left(\left(\mu_{2} \succ S_{n+k}\right) \times \mathrm{GL}(V)\right) / \mu_{2}}-->\frac{\operatorname{Mat}_{(n+k) \times(n+k)}^{\mathrm{sym}}(\mathbb{C}) \times \mathrm{Gr}_{k}\left(\mathbb{C}^{n+k}\right)}{\mathrm{PO}_{n+k}} .
$$

The symmetric matrices in question correspond to endomorphisms of $\mathbb{C}^{n+k}$ which are self-adjoint for the standard bilinear form. It follows that eigenvectors with different eigenvalues are orthogonal. A generic self-adjoint endomorphism has no multiple eigenvalues and its eigenvectors are not isotropic. Hence, it admits an orthonormal basis consisting of eigenvectors, which is unique up to permutation and signs. This means that the rational map (29) is indeed birational, so

$$
\frac{R_{n, k}^{0}}{G_{n, k}} \simeq \frac{\operatorname{Mat}_{(n+k) \times(n+k)}^{\mathrm{sym}}(\mathbb{C}) \times \mathrm{Gr}_{k}\left(\mathbb{C}^{n+k}\right)}{\mathrm{PO}_{n+k}} \times \mathbb{C}^{(4 n-1)(n+k)} .
$$

Now, the theorem is a consequence of the following proposition. 


\section{Costa, N. Hoffmann, R.M. Miró-Roig, A. Schmitt}

Proposition 4.11. Suppose that $d=d_{1}+d_{2}$ with $d_{1}, d_{2} \geqslant 1$, and $\nu \in\{1,2\}$. Then,

$$
\frac{\operatorname{Mat}_{d \times d}^{\mathrm{sym}}(\mathbb{C}) \times \operatorname{Gr}_{d_{\nu}}\left(\mathbb{C}^{d}\right)}{\mathrm{PO}_{d}} \simeq \frac{\operatorname{Mat}_{2^{e} \times 2^{e}}^{\mathrm{sym}}(\mathbb{C})^{2}}{\mathrm{PO}_{2^{e}}} \times \mathbb{C}^{d+d_{1} d_{2}-2^{e}\left(2^{e}+3\right) / 2},
$$

where $2^{e}$ is the largest power of 2 that divides both $d_{1}$ and $d_{2}$.

Proof. We endow $\mathbb{C}^{d}$ with the standard symmetric bilinear form $b_{d}: \mathbb{C}^{d} \times \mathbb{C}^{d} \longrightarrow \mathbb{C}$. Fix $\nu=1$ or $\nu=2$. Let a general linear subspace $U_{\nu} \subseteq \mathbb{C}^{d}$ of dimension $d_{\nu}$ be given. Then, $U_{3-\nu}:=U_{\nu}^{\perp}$ has dimension $d_{3-\nu}$, and

$$
\mathbb{C}^{d}=U_{1} \oplus U_{2}
$$

Let moreover $f: \mathbb{C}^{d} \longrightarrow \mathbb{C}^{d}$ be a generic self-adjoint endomorphism. With respect to the above orthogonal direct sum decomposition, we write

$$
f=\left(\begin{array}{ll}
f_{11} & f_{12} \\
f_{21} & f_{22}
\end{array}\right): U_{1} \oplus U_{2} \longrightarrow U_{1} \oplus U_{2}
$$

The self-adjointness $f=f^{*}$ means that $f_{11}=f_{11}^{*}, f_{22}=f_{22}^{*}$, and $f_{21}=f_{12}^{*}$. We may assume that $d_{1} \geqslant d_{2}$ without loss of generality. Since $f$ is generic, $f_{12}: U_{2} \longrightarrow U_{1}$ is then injective. We choose an orthonormal basis of $U_{1}$.

In the case $d_{1}>d_{2}$, we define a dominant rational map

$$
\frac{\mathrm{Mat}_{d \times d}^{\mathrm{sym}}(\mathbb{C}) \times \mathrm{Gr}_{d_{\nu}}\left(\mathbb{C}^{d}\right)}{\mathrm{PO}_{d}}-\rightarrow \frac{\mathrm{Mat}_{d_{1} \times d_{1}}^{\mathrm{sym}}(\mathbb{C}) \times \mathrm{Gr}_{d_{2}}\left(\mathbb{C}^{d_{1}}\right)}{\mathrm{PO}_{d_{1}}},
$$

by sending the pair $\left(f, U_{\nu}\right)$ to $f_{11}: U_{1} \longrightarrow U_{1}$ and $U_{2}^{\prime}:=f_{12}\left(U_{2}\right) \subseteq U_{1}$, where we identify $U_{1}$ with $\mathbb{C}^{d_{1}}$ via the chosen basis; this is well defined modulo $\mathrm{PO}_{d_{1}}$.

Using the isomorphism $f_{12}: U_{2} \longrightarrow U_{2}^{\prime}$, the restriction of $b_{d}$ to $U_{2} \times U_{2}$ and the map $f_{22}: U_{2} \longrightarrow U_{2}$ yield a nondegenerate symmetric bilinear form on $U_{2}^{\prime}$ and a self-adjoint endomorphism $f_{22}^{\prime}$ of $U_{2}^{\prime}$. From all these, we can reconstruct $f$ up to $\mathrm{PO}_{d}$ by choosing an orthogonal isomorphism $U_{1} \oplus U_{2}^{\prime} \cong \mathbb{C}^{d}$.

Given a generic point $\left(f_{11}: U_{1} \longrightarrow U_{1}, U_{2}^{\prime} \subseteq U_{1}\right)$ in the image of our rational map (30), we have just seen that the fiber over it parameterizes nondegenerate symmetric bilinear forms on $U_{2}^{\prime}$ together with self-adjoint endomorphisms $f_{22}^{\prime}$ of $U_{2}^{\prime}$. Since $\mu_{2} \subseteq \mathrm{O}_{d_{1}}$ acts by its nontrivial character on $U_{2}^{\prime} \subseteq U_{1}$, it acts trivially on these forms and endomorphisms; hence, the rational map (30) is birationally a tower of two vector bundles, both of rank $d_{2} \cdot\left(d_{2}+1\right) / 2$. This proves that

$$
\frac{\operatorname{Mat}_{d \times d}^{\mathrm{sym}}(\mathbb{C}) \times \mathrm{Gr}_{d_{\nu}}\left(\mathbb{C}^{d}\right)}{\mathrm{PO}_{d}} \simeq \frac{\operatorname{Mat}_{d_{1} \times d_{1}}^{\mathrm{sym}}(\mathbb{C}) \times \mathrm{Gr}_{d_{2}}\left(\mathbb{C}^{d_{1}}\right)}{\mathrm{PO}_{d_{1}}} \times \mathbb{C}^{d_{2}\left(d_{2}+1\right)},
$$

for $\nu=1$ and for $\nu=2$; recall that we are assuming that $d=d_{1}+d_{2}$ with $d_{1}>d_{2}$.

In the case $d_{1}=d_{2}$, genericity of $f$ implies that $f_{21}: U_{1} \longrightarrow U_{2}$ is an isomorphism. We define a dominant rational map

$$
\frac{\mathrm{Mat}_{d \times d}^{\mathrm{sym}}(\mathbb{C}) \times \mathrm{Gr}_{d_{1}}\left(\mathbb{C}^{d}\right)}{\mathrm{PO}_{d}}-\rightarrow \frac{\mathrm{Mat}_{d_{1} \times d_{1}}^{\mathrm{sym}}(\mathbb{C})^{2}}{\mathrm{PO}_{d_{1}}}
$$

by sending $\left(f, U_{1}\right)$ to $f_{11}: U_{1} \longrightarrow U_{1}$ and $f_{21}^{*}\left(b_{d}\right): U_{1} \times U_{1} \longrightarrow \mathbb{C}$, which correspond to symmetric matrices via the chosen basis of $U_{1}$; this is well defined modulo $\mathrm{PO}_{d_{1}}$.

The endomorphism $f_{22}^{\prime}:=f_{21}^{-1} \circ f_{22} \circ f_{21}$ of $U_{1}$ is self-adjoint with respect to $f_{21}^{*}\left(b_{d}\right)$. From all these, we can again reconstruct $f$ up to $\mathrm{PO}_{d}$. Since $\mu_{2} \subseteq \mathrm{O}_{d_{1}}$ acts trivially on these endo- 


\section{RATional FAMilies of InStANTON BUNDLES ON $\mathbb{P}^{2 n+1}$}

morphisms $f_{22}^{\prime}$, the rational map (32) is birationally a vector bundle of rank $d_{1} \cdot\left(d_{1}+1\right) / 2$. This proves that

$$
\frac{\mathrm{Mat}_{d \times d}^{\mathrm{sym}}(\mathbb{C}) \times \mathrm{Gr}_{d_{1}}\left(\mathbb{C}^{d}\right)}{\mathrm{PO}_{d}} \simeq \frac{\mathrm{Mat}_{d_{1} \times d_{1}}^{\mathrm{sym}}(\mathbb{C})^{2}}{\mathrm{PO}_{d_{1}}} \times \mathbb{C}^{d_{1}\left(d_{1}+1\right) / 2}
$$

under the assumption $d=d_{1}+d_{2}$ with $d_{1}=d_{2}$.

Now, let's return to the general case $d=d_{1}+d_{2}$ with $d_{1}, d_{2} \geqslant 1$. Following the Euclidean algorithm as it computes $h:=\operatorname{gcd}\left(d_{1}, d_{2}\right)$, and composing with the corresponding birational equivalence (31) or (33) in each step, we get

$$
\frac{\operatorname{Mat}_{d \times d}^{\mathrm{sym}}(\mathbb{C}) \times \mathrm{Gr}_{d_{\nu}}\left(\mathbb{C}^{d}\right)}{\mathrm{PO}_{d}} \simeq \frac{\mathrm{Mat}_{h \times h}^{\mathrm{sym}}(\mathbb{C})^{2}}{\mathrm{PO}_{h}} \times \mathbb{C}^{d+d_{1} d_{2}-h(h+3) / 2} .
$$

Recall that $2^{e}$ is the largest power of 2 dividing $h$. If $h=2^{e}$, then we are done, so we assume that $2^{e}<h$. Since the action of $\mathrm{PO}_{h}$ is generically free here, the stack quotient $\left[\mathrm{Mat}_{h \times h}^{\mathrm{sym}}(\mathbb{C})^{2} / \mathrm{O}_{h}\right]$ is generically a $\mu_{2}$-gerbe over our birational quotient modulo $\mathrm{PO}_{h}$. The standard representation $\mathbb{C}^{h}$ of $\mathrm{O}_{h}$ yields a vector bundle of rank $h$ and nontrivial weight on this $\mu_{2}$-gerbe, whose index (at the generic point) therefore divides $h$, and, hence, divides $2^{e}$. It follows that the Grassmannian bundle with fibers $\mathrm{Gr}_{2}\left(\mathbb{C}^{h}\right)$ over this stack quotient has a rational generic fiber, so

$$
\frac{\mathrm{Mat}_{h \times h}^{\mathrm{sym}}(\mathbb{C})^{2}}{\mathrm{PO}_{h}} \times \mathbb{C}^{2^{e}\left(h-2^{e}\right)} \simeq \frac{\mathrm{Mat}_{h \times h}^{\mathrm{sym}}(\mathbb{C})^{2} \times \mathrm{Gr}_{2^{e}}\left(\mathbb{C}^{h}\right)}{\mathrm{PO}_{h}} .
$$

Applying (34) once more, with $h$ and $2^{e}$ instead of $d$ and $d_{\nu}$, completes the proof.

Remark 4.12. The same argument shows that the stack quotient $\left[R_{n, k}^{0} / G_{n, k}\right]$ is birational to $\mathbb{C}^{5 k n+4 n^{2}-2^{e}\left(2^{e}+3\right) / 2} \times\left[\mathrm{Mat}_{2^{e} \times 2^{e}}^{\mathrm{sym}}(\mathbb{C})^{2} / \mathrm{O}_{2^{e}}\right]$. In particular, there is a Poincaré family on some dense open part if and only if $2^{e}=1$, which means that $n$ or $k$ is odd. Otherwise, the obstruction is a Brauer class of order 2 and index $2^{e}$; see [BCD12] and [Ami72], Theorem 3.

\section{The moduli space of RS-instanton bundles and its birational type}

In this section, we construct the moduli stack of RS-instanton bundles on $\mathbb{P}$ as well as the moduli space of stable RS-instanton bundles, and we determine the birational type of these objects. As before, the moduli spaces are obtained as quotients of a parameter space by a group action. This time the group that acts is nonreductive.

Put $U:=\mathbb{C}^{2}$, and let $p, q \geqslant 1$ be integers. We consider the multiplication map

$$
\mu: S^{p} U \otimes S^{q} U \longrightarrow S^{p+q} U .
$$

Lemma 5.1. For a linear hyperplane $\wp \subset S^{p} U$, the following are equivalent.

(i) There is a line $\ell \subset U$ with $\wp=\ell \cdot S^{p-1} U$.

(ii) The restriction $\wp \otimes S^{q} U \longrightarrow S^{p+q} U$ of $\mu$ is not surjective.

Proof. The case $p=1$ is trivial, so we assume that $p \geqslant 2$. It is obvious that statement (i) implies statement (ii).

For the converse, we assume that there is no line $\ell \subset U$ with $\wp=\ell \cdot S^{p-1} U$. We identify $U$ with $H^{0}\left(\mathbb{P}^{1}, \mathscr{O}(1)\right)$; then

$$
\wp \subset S^{p} U=H^{0}\left(\mathbb{P}^{1}, \mathscr{O}(p)\right)
$$




\section{Costa, N. Hoffmann, R.M. Miró-Roig, A. Schmitt}

The assumption on $\wp$ means that the canonical evaluation map

$$
\eta: \mathscr{O}_{\mathbb{P}^{1}}^{p} \cong \wp \otimes_{\mathbb{C}} \mathscr{O}_{\mathbb{P}^{1}} \longrightarrow \mathscr{O}_{\mathbb{P}^{1}}(p)
$$

is surjective. The kernel of $\eta$ is a vector bundle of rank $p-1$ over $\mathbb{P}^{1}$, so

$$
\operatorname{ker}(\eta) \cong \bigoplus_{i=1}^{p-1} \mathscr{O}_{\mathbb{P}^{1}}\left(a_{i}\right) \quad \text { with } a_{1}, \ldots, a_{p-1} \in \mathbb{Z}
$$

due to Grothendieck's splitting theorem. We have a short exact sequence

$$
0 \longrightarrow \operatorname{ker}(\eta) \longrightarrow \wp \otimes_{\mathbb{C}} \mathscr{O}_{\mathbb{P}^{1}} \stackrel{\eta}{\longrightarrow} \mathscr{O}_{\mathbb{P}^{1}}(p) \longrightarrow 0
$$

of vector bundles over $\mathbb{P}^{1}$. The associated long exact cohomology sequence reads

$$
0 \longrightarrow H^{0}\left(\mathbb{P}^{1}, \operatorname{ker}(\eta)\right) \longrightarrow \wp \longrightarrow S^{p} U \longrightarrow H^{1}\left(\mathbb{P}^{1}, \operatorname{ker}(\eta)\right) \longrightarrow 0
$$

Hence, we conclude that $H^{0}\left(\mathbb{P}^{1}, \operatorname{ker}(\eta)\right)=0$ and $H^{1}\left(\mathbb{P}^{1}, \operatorname{ker}(\eta)\right) \cong \mathbb{C}$. Comparing this with the decomposition (35), we see that $\operatorname{ker}(\eta) \cong \mathscr{O}_{\mathbb{P}^{1}}(-1)^{p-2} \oplus \mathscr{O}_{\mathbb{P}^{1}}(-2)$ and, in particular,

$$
H^{1}\left(\mathbb{P}^{1}, \operatorname{ker}(\eta) \otimes \mathscr{O}_{\mathbb{P}^{1}}(q)\right)=0 .
$$

This implies that the following map induced by $\eta$ is surjective:

$$
H^{0}\left(\mathbb{P}^{1}, \wp \otimes_{\mathbb{C}} \mathscr{O}_{\mathbb{P}^{1}}(q)\right) \longrightarrow H^{0}\left(\mathbb{P}^{1}, \mathscr{O}_{\mathbb{P}^{1}}(p+q)\right) .
$$

But this map is the restriction of the multiplication map $\mu$; therefore this restriction is surjective. This shows that statement (ii) is false if statement (i) is false.

Corollary 5.2. Suppose that three linear maps

$$
\alpha \in \mathrm{GL}\left(S^{p} U\right), \quad \beta \in \mathrm{GL}\left(S^{q} U\right), \quad \text { and } \quad \gamma \in \mathrm{GL}\left(S^{p+q} U\right)
$$

satisfy $\mu \circ(\alpha \otimes \beta)=\gamma \circ \mu$. Then, there is a linear map $g \in \operatorname{GL}(U)$ such that

$$
S^{p} g \in \mathbb{C}^{*} \cdot \alpha, \quad S^{q} g \in \mathbb{C}^{*} \cdot \beta, \quad \text { and } \quad S^{p+q} g \in \mathbb{C}^{*} \cdot \gamma .
$$

This linear map $g$ is unique up to multiplication by $\mathbb{C}^{*}$.

Proof. The map

$$
\begin{aligned}
\mathbb{P}(U) & \longrightarrow \mathbb{P}\left(S^{p} U\right)^{*} \\
\ell & \longmapsto \ell \cdot S^{p-1} U
\end{aligned}
$$

is a closed immersion. Its image is stable under the automorphism of $\mathbb{P}\left(S^{p} U\right)^{*}$ induced by $\alpha$, according to the previous lemma. Thus, $\alpha$ induces an automorphism of $\mathbb{P}(U)$, which we lift to an automorphism $g$ of $U$. Modifying $\alpha, \beta$, and $\gamma$ by $S^{p} g, S^{q} g$, and $S^{p+q} g$, respectively, we may assume that $g=\mathrm{id}_{U}$. This means that the automorphism of $\mathbb{P}\left(S^{p} U\right)^{*}$ induced by $\alpha$ restricts to the identity on $\mathbb{P}(U) \subseteq \mathbb{P}\left(S^{p} U\right)^{*}$.

Given a line $\ell$ in $U$, the hyperplane $\wp:=\ell \cdot S^{q-1} U$ in $S^{q} U$ has the property that $\left(\ell \cdot S^{p-1} U\right) \otimes$ $S^{q} U$ and $S^{p} U \otimes \wp$ have the same image under $\mu$. According to Corollary 5.2, this characterizes $\wp$ uniquely. Since $\alpha\left(\ell \cdot S^{p-1} U\right)=\ell \cdot S^{p-1} U$ due to the previous paragraph, we conclude that $\beta\left(\ell \cdot S^{q-1} U\right)=\ell \cdot S^{q-1} U$ as well.

The fundamental theorem of algebra states that every line $\ell$ in $S^{p} U$ is a product of $p$ lines $\ell_{1}, \ldots, \ell_{p}$ in $U$. If $\ell$ is generic, then the $\ell_{i}$ are all distinct, and $\ell$ is the intersection of the hyperplanes $\wp_{i}=\ell_{i} \cdot S^{p-1} U$ in $S^{p} U$. Due to the first paragraph, we have $\alpha\left(\wp_{i}\right)=\wp_{i}$ for all $i$, and, 


\section{RATional FAMilies of Instanton BUndLES ON $\mathbb{P}^{2 n+1}$}

hence, $\alpha(\ell)=\ell$ for generic lines $\ell$. It follows that $\alpha$ induces the identity on $\mathbb{P}\left(S^{p} U\right)$, and, hence, that $\alpha \in \mathbb{C}^{*} \cdot$ id.

Applying the same arguments to $\beta$, we get $\beta \in \mathbb{C}^{*}$. id as well. Because $\mu$ is surjective, this implies that $\gamma \in \mathbb{C}^{*} \cdot \mathrm{id}$, too.

\section{Let}

$$
f:\left(S^{n} U\right)^{*} \longrightarrow H^{0}\left(\mathbb{P}, \mathscr{O}_{\mathbb{P}}(1)\right)
$$

be a linear embedding. We fix an integer $k \geqslant 1$ and consider a linear map

$$
h: S^{n+2 k-2} U \longrightarrow H^{0}\left(\mathbb{P}, \mathscr{O}_{\mathbb{P}}(1)\right) .
$$

We endow the trivial algebraic vector bundle of rank $2 n+2 k$ over $\mathbb{P}$,

$$
E_{n, k}^{0}:=\left(\left(S^{n+k-1} U\right)^{*} \oplus S^{n+k-1} U\right) \otimes_{\mathbb{C}} \mathscr{O}_{\mathbb{P}},
$$

with the standard symplectic form $J$. We also form the algebraic vector bundles

$$
E_{n, k}^{-1}:=\left(S^{k-1} U\right) \otimes_{\mathbb{C}} \mathscr{O}_{\mathbb{P}}(-1) \quad \text { and } \quad E_{n, k}^{1}:=\left(S^{k-1} U\right)^{*} \otimes_{\mathbb{C}} \mathscr{O}_{\mathbb{P}}(1)
$$

of rank $k$ over $\mathbb{P}$. The linear maps $f$ and $h$ define a morphism of vector bundles

$$
A_{f, h}: E_{n, k}^{0} \longrightarrow E_{n, k}^{1}
$$

whose induced map of global sections is the direct sum of the following two maps:

$$
\begin{aligned}
& \left(S^{n+k-1} U\right)^{*} \stackrel{\mu^{*}}{\longrightarrow}\left(S^{k-1} U\right)^{*} \otimes\left(S^{n} U\right)^{*} \stackrel{\operatorname{id} \otimes f}{\longrightarrow}\left(S^{k-1} U\right)^{*} \otimes H^{0}\left(\mathbb{P}, \mathscr{O}_{\mathbb{P}}(1)\right), \\
& S^{n+k-1} U \stackrel{\mu_{*}}{\longrightarrow}\left(S^{k-1} U\right)^{*} \otimes S^{n+2 k-2} U \stackrel{\text { id } \otimes h}{\longrightarrow}\left(S^{k-1} U\right)^{*} \otimes H^{0}\left(\mathbb{P}, \mathscr{O}_{\mathbb{P}}(1)\right) .
\end{aligned}
$$

We assume that $A_{f, h}$ is surjective; this is an open condition on $h$. The composition

$$
E_{n, k}^{-1} \stackrel{J \circ A_{f, h}^{*}}{\longrightarrow} E_{n, k}^{0} \stackrel{A_{f, h}}{\longrightarrow} E_{n, k}^{1}
$$

vanishes; this follows easily from the observation that the bilinear map

$$
S^{k-1} U \otimes S^{k-1} U \stackrel{\mu}{\longrightarrow}\left(S^{n} U\right)^{*} \otimes S^{n+2 k-2} U \stackrel{f \otimes h}{\longrightarrow} H^{0}\left(\mathbb{P}, \mathscr{O}_{\mathbb{P}}(2)\right)
$$

is symmetric. Thus, for a generic choice of $h,(38)$ is a monad and defines a symplectic instanton bundle $E$.

The special form of the map $A_{f, h}$ means precisely that $E$ is an RS-instanton bundle. To see this, choose a basis of $U$, and endow the symmetric powers of $U$ and their duals with the induced bases. Let $f_{i}, h_{i} \in H^{0}\left(\mathbb{P}, \mathscr{O}_{\mathbb{P}}(1)\right)$ denote the images of these basis vectors under $f, h$. Then, the map (36) corresponds to the special matrix $F$ with entries $f_{i}$, and the map (37) corresponds to the persymmetric matrix $H$ with entries $h_{i}$. Moreover, for a generic choice of the $h_{i}$, the $n$-space $\left\{f_{0}=f_{1}=\cdots=f_{n}=0\right\}$ does not contain any zeros of the maximal minors of $H$, and, thus, we conclude that $E$ is an RS-instanton bundle.

Conversely, if $E$ is an RS-instanton bundle, then the entries of the matrices $F$ and $H$ define linear maps $f$ and $h$ as above. This shows that the RS-instanton bundles are precisely the instanton bundles arising from monads of the form (38).

Let $L_{f} \subseteq \mathbb{P}$ denote the locus where all sections in the image of $f$ vanish. Then, $L_{f}$ is a linear subspace of dimension $n$ in $\mathbb{P}$; it is the linear subspace restricted to which $E$ has $n+k$ global sections by construction. If $E$ is generic, then $L_{f}$ is the only linear subspace of dimension $n$ in $\mathbb{P}$ with that property; see Proposition 3.11. 


\section{Costa, N. Hoffmann, R.M. Miró-Roig, A. Schmitt}

The next question is: When are two such RS-instanton bundles isomorphic? Let

$$
g \in \operatorname{GL}(U), \quad t \in \mathbb{C}^{*}, \quad \text { and } \quad u \in\left(S^{2 n+2 k-2} U\right)^{*}
$$

be given. They define an isomorphism of symplectic monads

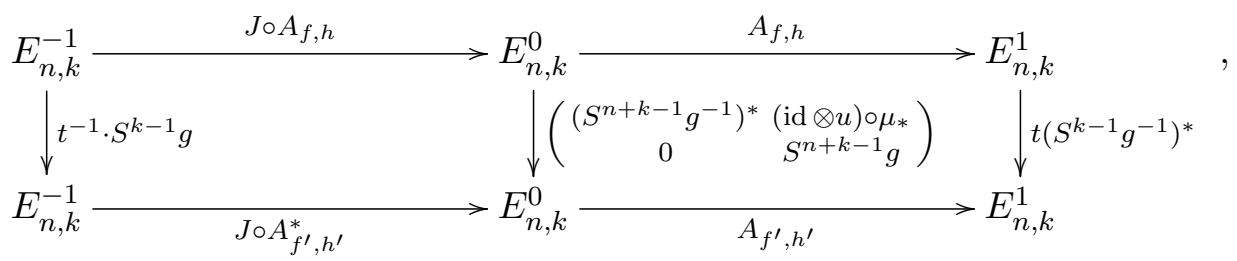

where the vertical map in the middle contains as a matrix entry the composition

$$
S^{n+k-1} U \stackrel{\mu_{*}}{\longrightarrow}\left(S^{n+k-1} U\right)^{*} \otimes S^{2 n+2 k-2} U \stackrel{\operatorname{id} \otimes u}{\longrightarrow}\left(S^{n+k-1} U\right)^{*}
$$

and the monad in the second row comes from the maps

$$
f^{\prime}:=t f \circ\left(S^{n} g\right)^{*} \quad \text { and } \quad h^{\prime}:=t\left(h-(u \otimes f) \circ \mu_{*}\right) \circ\left(S^{n+2 k-2} g^{-1}\right) .
$$

Here, the definition of the map $h^{\prime}$ involves the composition

$$
S^{n+2 k-2} U \stackrel{\mu_{*}}{\longrightarrow} S^{2 n+2 k-2} U \otimes\left(S^{n} U\right)^{*} \stackrel{u \otimes f}{\longrightarrow} H^{0}\left(\mathbb{P}, \mathscr{O}_{\mathbb{P}}(1)\right) .
$$

A straightforward computation shows that Diagram (39) commutes indeed and that the induced isomorphism of RS-instanton bundles is symplectic. Note that $L_{f}=L_{f^{\prime}}$.

The formulas (40) define a linear group action of the semidirect product

$$
G:=\left(\mathrm{GL}(U) \times \mathbb{C}^{*}\right) \ltimes\left(S^{2 n+2 k-2} U\right)^{*}
$$

on the vector space containing the pairs $(f, h)$ considered above, which is

$$
\mathrm{RS}:=\mathrm{RS}_{n, k}:=\left(S^{n} U \oplus\left(S^{n+2 k-2} U\right)^{*}\right) \otimes H^{0}\left(\mathbb{P}, \mathscr{O}_{\mathbb{P}}(1)\right),
$$

such that each group element sending $(f, h)$ to $\left(f^{\prime}, h^{\prime}\right)$ induces a symplectic isomorphism (39) from the monad given by $A_{f, h}$ to the monad given by $A_{f^{\prime}, h^{\prime}}$. This isomorphism is the identity if and only if the triple $(g, t, u)$ is in the image of the roots of unity $\mu_{n+k-1} \subseteq \mathbb{C}^{*}$ under the embedding

$$
\begin{aligned}
\mu_{n+k-1} & \longrightarrow\left(\mathrm{GL}(U) \times \mathbb{C}^{*}\right) \ltimes\left(S^{2 n+2 k-2} U\right)^{*} \\
\rho & \longmapsto\left(\rho \cdot \mathrm{id}_{U}, \rho^{-n}, 0\right) .
\end{aligned}
$$

This image is a normal subgroup, and the resulting quotient group

$$
G:=\frac{\left(\mathrm{GL}(U) \times \mathbb{C}^{*}\right) \ltimes\left(S^{2 n+2 k-2} U\right)^{*}}{\mu_{n+k-1}}
$$

still acts on the vector space $\mathrm{RS}$.

Proposition 5.3. Given two RS-instanton bundles

$$
E=\operatorname{ker}\left(A_{f, h}\right) / \operatorname{im}\left(J \circ A_{f, h}^{*}\right) \quad \text { and } \quad E^{\prime}=\operatorname{ker}\left(A_{f^{\prime}, h^{\prime}}\right) / \operatorname{im}\left(J \circ A_{f^{\prime}, h^{\prime}}^{*}\right)
$$

with $L_{f}=L_{f^{\prime}}$ and a symplectic isomorphism $\varphi: E \longrightarrow E^{\prime}$, there is a unique element of $G$ which sends $(f, h)$ to $\left(f^{\prime}, h^{\prime}\right)$ and gives back $\varphi$ via Diagram (39).

Proof. The uniqueness follows from the observation that the isomorphism (39) is the identity only if the triple $(g, t, u)$ is in the image of $\mu_{n+k-1}$. We prove the existence. 


\section{RATional FAMilies of Instanton BUndLES ON $\mathbb{P}^{2 n+1}$}

The given isomorphism $\varphi$ lifts uniquely to an isomorphism

$$
\varphi^{\bullet}:\left(E_{n, k}^{\bullet}, A_{f, h}\right) \stackrel{\sim}{\longrightarrow}\left(E_{n, k}^{\bullet}, A_{f^{\prime}, h^{\prime}}\right)
$$

of symplectic monads, which in turn is given by two linear automorphisms

$$
\varphi^{0} \in \operatorname{Sp}\left(\left(S^{n+k-1} U\right)^{*} \oplus S^{n+k-1} U, J\right) \quad \text { and } \quad \varphi^{1} \in \mathrm{GL}\left(\left(S^{k-1} U\right)^{*}\right)
$$

satisfying the following equation:

$$
A_{f^{\prime}, h^{\prime}} \circ \varphi^{0}=\varphi^{1} \circ A_{f, h} .
$$

Let's first restrict this equation to $L:=L_{f}=L_{f^{\prime}} \subset \mathbb{P}$. The first summand $\left(S^{n+k-1} U\right)^{*}$ of $H^{0}\left(\mathbb{P}, E_{n, k}^{0}\right)$ consists exactly of the sections that vanish on $L$. It follows that this summand has to be preserved by $\varphi^{0}$, so

$$
\varphi^{0}=\left(\begin{array}{cc}
\left(\gamma^{-1}\right)^{*} & \tau \\
0 & \gamma
\end{array}\right)
$$

for an appropriate linear automorphism $\gamma \in \mathrm{GL}\left(S^{n+k-1} U\right)$ and some symmetric bilinear form $\tau: S^{n+k-1} U \longrightarrow\left(S^{n+k-1} U\right)^{*}$.

The assumption $L_{f}=L_{f^{\prime}}$ means that the linear embeddings $f$ and $f^{\prime}$ have the same image, so there is a linear automorphism $\beta \in \mathrm{GL}\left(S^{n} U\right)$ with $f^{\prime}=f \circ \beta^{*}$. We can write $\varphi^{1}=\left(\alpha^{-1}\right)^{*}$ for a unique element $\alpha \in \mathrm{GL}\left(S^{k-1} U\right)$.

Now, the first component of (41) simplifies to $\mu \circ(\alpha \otimes \beta)=\gamma \circ \mu$. This allows us to apply Corollary 5.2. Multiplying the resulting element $g \in \mathrm{GL}(U)$ by a nonzero scalar, if necessary, we can even achieve $\gamma=S^{n+k-1} g$ in addition to $S^{k-1} g \in \mathbb{C}^{*} \cdot \alpha$ and $S^{n} g \in \mathbb{C}^{*} \cdot \beta$. Comparing the scalars, we get more precisely $\alpha=t^{-1} \cdot S^{k-1} g$ and $\beta=t \cdot S^{n} g$ for some $t \in \mathbb{C}^{*}$.

We replace the pair $(f, h)$ by its image under the group element given by the triple $(g, t, 0)$. This reduces us without loss of generality to the case where

$$
f=f^{\prime}, \quad \varphi^{0}=\left(\begin{array}{cc}
\mathrm{id} & \tau \\
0 & \mathrm{id}
\end{array}\right) \quad \text { and } \quad \varphi^{1}=\mathrm{id}
$$

for some symmetric bilinear form $\tau: S^{n+k-1} U \longrightarrow\left(S^{n+k-1} U\right)^{*}$. In this situation, the second component of (41) means that the diagram

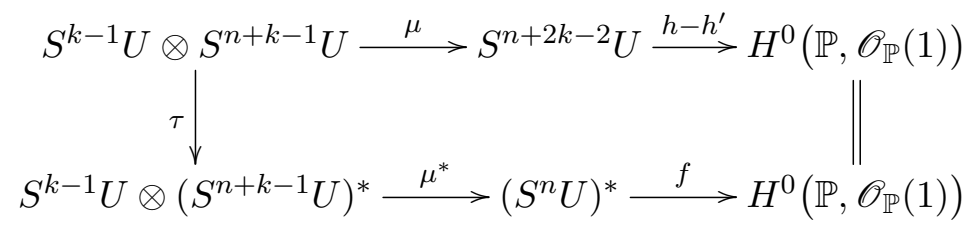

commutes. Since $\mu$ is surjective, we conclude that the image of $h-h^{\prime}$ is contained in the image of $f$. As $f$ is injective, this implies that $h-h^{\prime}=f \circ \delta$ for some linear map

$$
\delta: S^{n+2 k-2} U \longrightarrow\left(S^{n} U\right)^{*} .
$$

Using this, the previous commutative diagram yields the commutative diagram

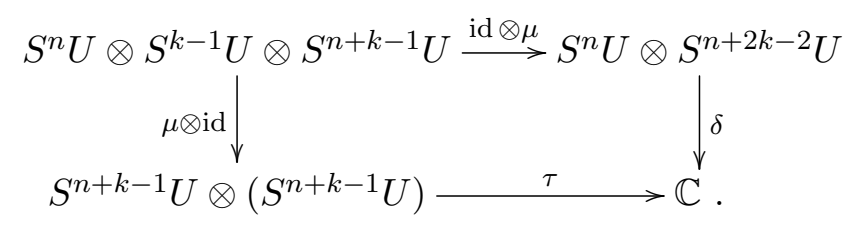




\section{Costa, N. Hoffmann, R.M. Miró-Roig, A. Schmitt}

The composition $\tau \circ(\mu \otimes \mathrm{id})=(\mathrm{id} \otimes \mu) \circ \delta$ in this diagram is a multilinear form

$$
U^{\otimes(2 n+2 k-2)}=U^{\otimes n} \otimes U^{\otimes(k-1)} \otimes U^{\otimes(n+k-1)} \longrightarrow \mathbb{C}
$$

which is invariant under the two subgroups $S_{n+k-1} \times S_{n+k-1}$ and $S_{n} \times S_{n+2 k-2}$ in the symmetric group $S_{2 n+2 k-2}$. But these two subgroups generate the full group $S_{2 n+2 k-2}$, so the multilinear form descends to a linear form

$$
u: S^{2 n+2 k-2} U \longrightarrow \mathbb{C}
$$

such that $\delta=u \circ \mu$ and $\tau=u \circ \mu$. It follows that the isomorphism $\varphi^{\bullet}$ comes from the element in $G$ given by the triple $\left(\operatorname{id}_{U}, 1, u\right)$.

Corollary 5.4. The quotient stack $[\mathrm{RS} / G]$ is birational to the moduli stack of $R S$-instanton bundles $E$ with charge $k$ over $\mathbb{P}^{2 n+1}$.

Remark 5.5. Note that the quotient stack $[\mathrm{RS} / G]$ has dimension

$$
\begin{aligned}
\operatorname{dim}(\mathrm{RS})-\operatorname{dim}(G) & =((n+1)+(n+2 k-1)) \cdot(2 n+2)-(4+1+(2 n+2 k-1)) \\
& =(4 n+2) \cdot k+4 n^{2}+2 n-4
\end{aligned}
$$

In the special case $n=1$, this coincides with the dimension $6 k+2$ of the moduli space introduced by Rao and by Skiti in [Rao97] and [Ski97], respectively.

Our next aim is to determine the birational type of this moduli stack. For that, we choose a basis of $H^{0}\left(\mathbb{P}, \mathscr{O}_{\mathbb{P}}(1)\right)$. This provides a $G$-equivariant isomorphism

$$
\mathrm{RS} \cong\left(S^{n} U\right)^{\oplus(2 n+2)} \oplus\left(\left(S^{n+2 k-2} U\right)^{*}\right)^{\oplus(2 n+2)} .
$$

We start with the quotient of the first summand $\left(S^{n} U\right)^{\oplus(2 n+2)}$ modulo the subgroup

$$
\left(\mathrm{GL}(U) \times \mathbb{C}^{*}\right) / \iota\left(\mu_{n+k-1}\right)
$$

of $G$, whose definition involves the embedding

$$
\begin{aligned}
\iota: \mathbb{C}^{*} & \longrightarrow \mathrm{GL}(U) \times \mathbb{C}^{*} \\
\lambda & \longmapsto\left(\lambda \cdot \operatorname{id}_{U}, \lambda^{-n}\right) .
\end{aligned}
$$

Here, the group action is the restriction of the action (40). So the group element represented by $g \in \mathrm{GL}(U)$ and $t \in \mathbb{C}^{*}$ acts on $\left(S^{n} U\right)^{\oplus(2 n+2)}$ as the automorphism

$$
\begin{aligned}
\left(S^{n} U\right)^{\oplus(2 n+2)} & \longrightarrow\left(S^{n} U\right)^{\oplus(2 n+2)} \\
f & \longmapsto\left(S^{n} g\right)(t f) .
\end{aligned}
$$

Proposition 5.6. The stack quotient of the vector space $\left(S^{n} U\right)^{\oplus(2 n+2)}$ modulo the above linear group action of $\left(\mathrm{GL}(U) \times \mathbb{C}^{*}\right) / \iota\left(\mu_{n+k-1}\right)$ is

(i) birational to $\mathbb{A}^{2 n^{2}+4 n-2} \times B \mathbb{C}^{*}$, if $n$ or $k$ is odd;

(ii) birational to $\mathbb{A}^{2 n^{2}+4 n-7} \times\left[\operatorname{End}(U)^{2} / \mathrm{GL}(U)\right]$, if $n$ and $k$ are even.

Here, $B \mathbb{C}^{*}$ is the classifying stack of $\mathbb{C}^{*}$, and $\left[\operatorname{End}(U)^{2} / \mathrm{GL}(U)\right]$ is the stack quotient of $\operatorname{End}(U)^{2}$ modulo the linear action of $\operatorname{GL}(U)$ by simultaneous conjugation.

Proof. We consider the central extension of linear algebraic groups

$$
1 \longrightarrow \mathbb{C}^{*} \cong \frac{\mathbb{C}^{*}}{\mu_{n+k-1}} \stackrel{\iota}{\longrightarrow} \frac{\mathrm{GL}(U) \times \mathbb{C}^{*}}{\iota\left(\mu_{n+k-1}\right)} \stackrel{\pi}{\longrightarrow} \frac{\mathrm{GL}(U)}{\mu_{n}} \longrightarrow 1,
$$




\section{RATional FAMilies of Instanton BUndLES ON $\mathbb{P}^{2 n+1}$}

where $\pi$ sends the class of a pair $(g, t)$ to the class of $t^{1 / n} \cdot g \in \mathrm{GL}(U)$; note that the $n$th root $t^{1 / n}$ is well defined modulo $\mu_{n}$. Our group action (42) is the composition of $\pi$ and the natural action of $\mathrm{GL}(U) / \mu_{n}$ on $\left(S^{n} U\right)^{\oplus(2 n+2)}$ via $n$th symmetric powers.

Suppose that $n$ is odd. Then, the central extension (43) splits, because the map

$$
\begin{aligned}
\frac{\mathrm{GL}(U) \times \mathbb{C}^{*}}{\iota\left(\mu_{n+k-1}\right)} & \longrightarrow \frac{\mathbb{C}^{*}}{\mu_{n+k-1}} \\
{[g, t] } & \longmapsto\left[t \cdot \operatorname{det}(g)^{\frac{n+1}{2}}\right]
\end{aligned}
$$

is a left inverse of $\iota$. Hence, the stack quotient in question is birational to $B \mathbb{C}^{*}$ times the stack quotient of $\left(S^{n} U\right)^{\oplus(2 n+2)}$ modulo $\mathrm{GL}(U) / \mu_{n}$. Note that already the action of $\mathrm{GL}(U) / \mu_{n}$ on $\left(S^{n} U\right)^{\oplus 2}$ is generically free. Moreover, the isomorphism

$$
\begin{aligned}
\mathrm{GL}(U) / \mu_{n} & \stackrel{\sim}{\longrightarrow} \mathrm{GL}(U) \\
{[g] } & \longmapsto \operatorname{det}(g)^{\frac{n-1}{2}} \cdot g
\end{aligned}
$$

shows that $\mathrm{GL}(U) / \mu_{n}$ also has a generically free representation of dimension four containing an open orbit. The no-name lemma ([BK86], Lemma 1.2) allows us to replace the representation $\left(S^{n} U\right)^{\oplus(2 n+2)}$ by one which contains this four-dimensional representation as a direct summand, and then to conclude that the quotient is rational, as required.

Now, suppose that $n$ is even and $k$ is odd. Then, (43) also splits, since the map

$$
\begin{aligned}
\frac{\mathrm{GL}(U) \times \mathbb{C}^{*}}{\iota\left(\mu_{n+k-1}\right)} & \longrightarrow \frac{\mathbb{C}^{*}}{\mu_{n+k-1}} \\
{[g, t] } & \longmapsto\left[\operatorname{det}(g)^{\frac{n+k-1}{2}}\right]
\end{aligned}
$$

is a left inverse of $\iota$. Hence, the stack quotient in question is again birational to $B \mathbb{C}^{*}$ times the stack quotient of $\left(S^{n} U\right)^{\oplus(2 n+2)}$ modulo $\mathrm{GL}(U) / \mu_{n}$. The isomorphism

$$
\begin{aligned}
\mathrm{GL}(U) / \mu_{n} & \stackrel{\sim}{\longrightarrow} \operatorname{PGL}(U) \times \mathbb{C}^{*} \\
{[g] } & \left.\longmapsto\left[g, \operatorname{det}(g)^{\frac{n}{2}}\right)\right]
\end{aligned}
$$

provides a generically free action of $\operatorname{GL}(U) / \mu_{n}$ on $\operatorname{End}(U)^{2} \oplus \mathbb{C}$, so that the quotient is rational. Using the no-name lemma as before, it follows that the quotient of $\left(S^{n} U\right)^{\oplus(2 n+2)}$ modulo $\mathrm{GL}(U) / \mu_{n}$ is also rational, as required.

Finally, suppose that $n$ and $k$ are both even. We use the group isomorphism

$$
\begin{aligned}
\frac{\mathrm{GL}(U) \times \mathbb{C}^{*}}{\iota\left(\mu_{n+k-1}\right)} \stackrel{\sim}{\longrightarrow} \mathrm{GL}(U) \times \mathbb{C}^{*} \\
{[g, t] \longmapsto\left(\operatorname{det}(g)^{\frac{n+k-2}{2}} \cdot g, \operatorname{det}(g)^{\frac{n}{2}} \cdot t\right), }
\end{aligned}
$$

whose composition with the embedding $\iota$ of $\mathbb{C}^{*} \cong \mathbb{C}^{*} / \mu_{n+k-1}$ is the embedding

$$
\begin{aligned}
\mathbb{C}^{*} & \longrightarrow \mathrm{GL}(U) \times \mathbb{C}^{*} \\
\lambda & \longmapsto\left(\lambda \cdot \operatorname{id}_{U}, 1\right) .
\end{aligned}
$$

Thus, the cokernel of $\iota$ is isomorphic to $\operatorname{PGL}(U) \times \mathbb{C}^{*}$, which again acts generically freely on $\operatorname{End}(U)^{2} \oplus \mathbb{C}$. The no-name lemma allows us to replace the representation $\left(S^{n} U\right)^{\oplus(2 n+2)}$ by the direct sum of $\operatorname{End}(U)^{2} \oplus \mathbb{C}$ and a trivial representation of dimension

$$
(n+1) \cdot(2 n+2)-2 \cdot 4-1=2 n^{2}+4 n-7,
$$




\section{Costa, N. Hoffmann, R.M. Miró-Roig, A. Schmitt}

whose quotient modulo $\mathrm{GL}(U) \times \mathbb{C}^{*}$ is precisely as claimed.

COROLlary 5.7. The moduli stack of RS-instanton bundles with charge $k$ over $\mathbb{P}^{2 n+1}$ is

(i) birational to $\mathbb{A}^{(4 n+2) k+4 n^{2}+2 n-4} \times B \mu_{2}$, if $n$ or $k$ is odd;

(ii) birational to $\mathbb{A}^{(4 n+2) k+4 n^{2}+2 n-9} \times\left[\operatorname{End}(U)^{2} / \operatorname{SL}(U)\right]$, if $n$ and $k$ are even.

Proof. The projection onto the first summand

$$
\mathrm{RS} \cong\left(S^{n} U\right)^{\oplus(2 n+2)} \oplus\left(\left(S^{n+2 k-2} U\right)^{*}\right)^{\oplus(2 n+2)} \longrightarrow\left(S^{n} U\right)^{\oplus(2 n+2)}
$$

descends to a 1-morphism of stacks

$$
\Phi:\left[\frac{\mathrm{RS}}{G}\right] \longrightarrow\left[\frac{\left(S^{n} U\right)^{\oplus(2 n+2)}}{\left(\mathrm{GL}(U) \times \mathbb{C}^{*}\right) / \iota\left(\mu_{n+k-1}\right)}\right] .
$$

The latter stack is generically a gerbe with band $\mathbb{C}^{*}$ due to the previous proposition. The 1 -morphism $\Phi$ is a vector bundle $\mathscr{V}$ with fibers

$$
\left(\left(S^{n+2 k-2} U\right)^{*}\right)^{\oplus(2 n+2)} / f_{*}\left(\left(S^{2 n+2 k-2} U\right)^{*}\right),
$$

on which the automorphism groups $\mathbb{C}^{*}$ of the gerbe act with weight 2 . Its rank is

$$
\operatorname{rank}(\mathscr{V})=(n+2 k-1) \cdot(2 n+2)-(2 n+2 k-1)=(4 n+2) \cdot k+2 n^{2}-2 n-1 .
$$

Now, suppose that $n$ or $k$ is odd. Let $\mathscr{L}$ denote the tensor square of the universal line bundle over the classifying stack $B \mathbb{C}^{*}$. Then, the automorphism groups $\mathbb{C}^{*}$ of this neutral gerbe $B \mathbb{C}^{*}$ act with the same weight two on the fibers of $\mathscr{L}$. But any two vector bundles over a $\mathbb{C}^{*}$-gerbe with the same rank and the same weight are isomorphic over some dense open substack of that $\mathbb{C}^{*}$-gerbe, according to [Hof07], Lemma 4.10. Using Proposition 5.6, we conclude that the total space $[\mathrm{RS} / G]$ of $\mathscr{V}$ is birational to $\mathbb{A}^{2 n^{2}+4 n-2}$ times the total space of the vector bundle

$$
\mathscr{L}^{\oplus(4 n+2) k+2 n^{2}-2 n-1} \longrightarrow B \mathbb{C}^{*} .
$$

The projection of the line bundle $\mathscr{L}$ onto $B \mathbb{C}^{*}$ coincides with the natural morphism

$$
\varphi: B \mu_{2} \longrightarrow B \mathbb{C}^{*} \text {. }
$$

Hence, the total space of $\mathscr{L}^{\oplus N+1}$ over $B \mathbb{C}^{*}$ is the total space of $\varphi^{*} \mathscr{L}^{\oplus N}$ over $B \mu_{2}$. But this pullback vector bundle is trivial, because $\mu_{2} \subset \mathbb{C}^{*}$ acts trivially on the fibers of $\mathscr{L}$. This shows that the total space of $\mathscr{L}^{\oplus N+1}$ over $B \mathbb{C}^{*}$ is isomorphic to $\mathbb{A}^{N} \times B \mu_{2}$. It follows that the stack $[\mathrm{RS} / G]$ is birational to

$$
\mathbb{A}^{2 n^{2}+4 n-2} \times \mathbb{A}^{(4 n+2) k+2 n^{2}-2 n-2} \times B \mu_{2}
$$

in the case where $n$ or $k$ is odd.

Now, suppose that $n$ and $k$ are even. Let $\mathscr{U}$ denote the universal vector bundle of rank two over the quotient stack $\left[\operatorname{End}(U)^{2} / \mathrm{GL}(U)\right]$, or in other words the pullback of the universal vector bundle of rank two over the classifying stack $B \mathrm{GL}(U)$. On the line bundle $\operatorname{det}(\mathscr{U})$, the automorphism groups $\mathbb{C}^{*}$ of the dense open $\mathbb{C}^{*}$-gerbe in $\left[\operatorname{End}(U)^{2} / \mathrm{GL}(U)\right]$ act again with weight two. Combining [Hof07], Lemma 4.10, and Proposition 5.6 as before, we conclude that the stack $[\mathrm{RS} / G]$ is birational to $\mathbb{A}^{2 n^{2}+4 n-7}$ times the total space of the vector bundle

$$
\operatorname{det}(\mathscr{U})^{\oplus(4 n+2) k+2 n^{2}-2 n-1} \longrightarrow\left[\operatorname{End}(U)^{2} / \mathrm{GL}(U)\right] .
$$




\section{RATional FAMilies of InStANTON BUNDLES ON $\mathbb{P}^{2 n+1}$}

The projection of the line bundle $\operatorname{det}(\mathscr{U})$ onto $\left[\operatorname{End}(U)^{2} / \operatorname{GL}(U)\right]$ coincides with the natural morphism

$$
\psi:\left[\operatorname{End}(U)^{2} / \mathrm{SL}(U)\right] \longrightarrow\left[\operatorname{End}(U)^{2} / \mathrm{GL}(U)\right] .
$$

Hence, the total space of $\operatorname{det}(\mathscr{U})^{\oplus N+1}$ over $\left[\operatorname{End}(U)^{2} / \mathrm{GL}(U)\right]$ is the total space of $\psi^{*} \operatorname{det}(\mathscr{U})^{\oplus N}$ over $\left[\operatorname{End}(U)^{2} / \mathrm{SL}(U)\right]$. But this pullback vector bundle is now trivial over a dense open substack of $\left[\operatorname{End}(U)^{2} / \mathrm{SL}(U)\right]$, because the generic stabilizer $\mu_{2} \subset \mathrm{SL}(U)$ acts trivially on the fibers of $\operatorname{det}(\mathscr{U})$; see [Hof07], Corollary 4.8. This shows that the total space of $\operatorname{det}(\mathscr{U})^{\oplus N+1}$ over $\left[\operatorname{End}(U)^{2} / \mathrm{GL}(U)\right]$ is birational to $\mathbb{A}^{N} \times\left[\operatorname{End}(U)^{2} / \mathrm{SL}(U)\right]$. It follows that the stack $[\mathrm{RS} / G]$ is birational to

$$
\mathbb{A}^{2 n^{2}+4 n-7} \times \mathbb{A}^{(4 n+2) k+2 n^{2}-2 n-2} \times\left[\operatorname{End}(U)^{2} / \mathrm{SL}(U)\right]
$$

in the case where $n$ and $k$ are both even.

Next, we would like to construct the moduli space for Rao-Skiti instanton bundles as an algebraic variety. We have already set up the group action for this moduli problem. Since the group $G$ that acts is not reductive, it seems that the construction of the quotient is not as straightforward as in the setting of 't Hooft instanton bundles. However, we can easily reduce to a quotient problem for a reductive group. That problem can be solved with the construction of [OS86] and some descent theory. So, let us make all this precise. Denote by

$$
\mathrm{RS}_{n, k}^{\mathrm{s}} \subset \mathrm{RS}_{n, k}
$$

the $G$-invariant open subset of RS-monads as in (38) whose cohomology is a stable symplectic instanton bundle. By Remark 3.10 (i), this set is nonempty.

Proposition 5.8. For given positive integers $n$ and $k$, the moduli space

$$
\operatorname{RSI}_{\mathbb{P}^{2 n+1}}(k):=\mathrm{RS}_{n . k}^{\mathrm{s}} / G
$$

of stable $R S$-instanton bundles with charge $k$ on $\mathbb{P}^{2 n+1}$ exists as a smooth quasi-projective variety. It is equipped with a generically injective morphism

$$
\iota: \operatorname{RSI}_{\mathbb{P}^{2 n+1}}(k) \longrightarrow \mathrm{MI}_{\mathbb{P}^{2 n+1}}(k)
$$

to the moduli space of stable symplectic instanton bundles with charge $k$ on $\mathbb{P}^{2 n+1}$.

Proof. Formulas (36) and (37) describe an embedding

$$
\psi: \mathrm{RS}_{n, k} \longrightarrow \mathrm{SM}
$$

of $\mathrm{RS}_{n, k}$ into the space of all symplectic monads (compare (28)). We also have a homomorphism

$$
\alpha: G \longrightarrow H, \quad H:=\operatorname{Sp}\left(\left(S^{n+k-1} U\right)^{*} \oplus S^{n+k-1} U, J\right) \times \mathrm{GL}(V) .
$$

Note that $\alpha$ is injective (see Proposition 5.3 and its proof for the details).

The principal $G$-bundle $H \longrightarrow H / G$ and the action of $G$ on $\mathrm{RS}_{n, k}^{\mathrm{s}}$ define the associated fiber bundle

$$
H \stackrel{G}{\times} \mathrm{RS}_{n, k}^{\mathrm{s}} \longrightarrow H / G
$$

with typical fiber $\mathrm{RS}_{n, k}^{\mathrm{S}}$ (see [Ser08], $\S 3.2$ ). It is a smooth quasi-projective variety on which the reductive group $H$ acts from the left. By Proposition 3.11 and Proposition 5.3, we have a generically injective and $H$-equivariant morphism

$$
a: H \stackrel{G}{\times} \mathrm{RS}_{n, k}^{\mathrm{S}} \longrightarrow \mathrm{SM} .
$$




\section{Costa, N. Hoffmann, R.M. Miró-Roig, A. Schmitt}

Since $H \times{ }^{G} \mathrm{RS}_{n, k}^{\mathrm{s}}$ is normal, the $H$-action on this space can be linearized in some ample line bundle $A$ ([MFK94], Corollary 1.6).

Let

$$
\widehat{\mathrm{SI}}_{k} \subset \mathrm{SM} \backslash\{0\}
$$

be the preimage of $\widehat{\mathrm{SI}}_{k}$ under the projection $\mathrm{SM} \backslash\{0\} \rightarrow \mathbb{P}(\mathrm{SM})$. Remark 4.4 shows that

$$
\widehat{\mathrm{SI}}_{k} \longrightarrow \mathrm{MI}_{\mathbb{P}^{2 n+1}}(k)
$$

is a principal $H$-bundle. In particular, it is a faithfully flat morphism. The $H$-action on the space $H \times{ }^{G} \mathrm{RS}_{n, k}^{\mathrm{s}}$ and the linearization of this action in $A$ provide the data which enable us to descend the $H$-equivariant morphism

$$
H \stackrel{G}{\times} \mathrm{RS}_{n, k}^{\mathrm{s}} \longrightarrow \mathrm{SM}
$$

to a quasi-projective variety $Q$ and a morphism $Q \longrightarrow \mathrm{MI}_{\mathbb{P}^{2 n+1}}(k)$. For this, one applies a descent theorem by Grothendieck ([GR03], Exposé VIII, Proposition 7.8; [BLR90], Theorem 7, p. 138). Here,

$$
H \stackrel{G}{\times} \mathrm{RS}_{n, k}^{\mathrm{s}} \longrightarrow Q
$$

is also a principal $H$-bundle. In particular, $Q$ is the categorical quotient for $\operatorname{RS}_{n, k}^{\mathrm{s}}$ with respect to the $G$-action.

Proposition 5.6 implies the following statements.

Corollary 5.9. The coarse moduli space $\mathrm{RSI}_{\mathbb{P}^{2 n+1}}(k)$ of stable RS-instanton bundles with charge $k$ over $\mathbb{P}^{2 n+1}$ is rational.

Corollary 5.10. There is a Poincaré family parameterized by some open subscheme of the coarse moduli space $\operatorname{RSI}_{\mathbb{P}^{2 n+1}}(k)$ of stable $R S$-instanton bundles with charge $k$ over $\mathbb{P}^{2 n+1}$ if and only if $n$ is odd or $k$ is odd.

\section{REFERENCES}

Ami72 S.A. Amitsur, On central division algebras, Israel Jour. Math. 12 (1972), 408-420.

AO94 V. Ancona and G. Ottaviani, On the stability of special instanton bundles on $\mathbb{P}^{2 n+1}$, Trans. Amer. Math. Soc. 341 (1994), 677-693.

AO00 V. Ancona and G. Ottaviani, On the irreducible components of the moduli space of instanton bundles on $\mathbb{P}^{5}$, in Coen, Salvatore (ed.), Geometry seminars, 1998-1999, papers of a conference on real and complex analytic geometry, University of Bologna, Italy, November 1997, Bologna: Universit degli Studi di Bologna, Dipartimento di Matematica, 2000, 95-100.

AT77 M.F. Atiyah and R.S. Ward, Instantons and algebraic geometry, Comm. Math. Phys. 55 (1977), $117-124$.

Ati79 M.F. Atiyah, Geometry on Yang-Mills fields, Scuola Normale Superiore Pisa, Pisa, 1979.

BCD12 I. Biswas, E. Coskun, and A. Dhillon, The period-index problem of the canonical gerbe of symplectic and orthogonal bundles, arXiv:1009.3906v2 (2012).

Bei78 A.A. Beilinson, Coherent sheaves on $\mathbb{P}^{n}$ and problems in linear algebra, Funktsional. Anal. i Prilozhen 12 (1978), 68-69.

Ben05 E. Beneish, Centers of generic algebras with involution, J. Algebra 294 (2005), 41-50. http: //dx.doi.org/10.1016/j.jalgebra.2005.08.029 


\section{RATIONAL FAMILIES OF INSTANTON BUNDLES ON $\mathbb{P}^{2 n+1}$}

BK86 F.A. Bogomolov and P.I. Katsylo, Rationality of some quotient varieties. Math. USSR Sbornik 54 (1986), 571-576.

BLR90 S. Bosch, W. Lütkebohmert, and M. Raynaud, Néron models, Ergebnisse der Mathematik und ihrer Grenzgebiete, 3. Folge, vol. 21, Springer-Verlag, Berlin, 1990.

BV88 W. Bruns and U. Vetter, Determinantal rings, Lecture Notes in Mathematics, 1327, SpringerVerlag, Berlin, 1988.

CGK85 E. Corrigan, P. Goddard, and A. Kent, Some comments on the ADHM construction in $4 k$ dimensions, Comm. Math. Phys. 100 (1985), 1-13.

CO03 L. Costa and G. Ottaviani, Nondegenerate multidimensional matrices and instanton bundles, Trans. Amer. Math. Soc. 355 (2003), 49-55.

CS88 M.M. Capria and S.M. Salamon, Yang-Mills fields on quaternionic spaces, Nonlinearity 1 (1988), $517-530$.

GR03 A. Grothendieck and M. Raynaud, Revêtements étales et groupe fondamental (SGA 1), Séminaire de géométrie algébrique du Bois Marie 1960-61, updated and annotated reprint of the 1971 original, Documents Mathématiques, vol. 3, Société Mathématique de France, Paris, 2003.

GW09 R. Goodman and N.R. Wallach, Symmetry, representations, and invariants, Graduate Texts in Mathematics, 255, Springer, Dordrecht, 2009. http://dx.doi.org/10.1007/ 978-0-387-79852-3

HN82 A. Hirschowitz and M.S. Narasimhan, Fibrés de 't Hooft spéciaux et applications, Enumerative geometry and classical algebraic geometry (Nice, 1981), 143-164, Progr. Math., 24, Birkhäuser, Boston, Mass., 1982.

Hof07 N. Hoffmann, Rationality and Poincaré families for vector bundles with extra structure on a curve, Int. Math. Res. Not. Article ID rnm010 (2007). http://dx.doi.org/10.1093/imrn/ rnm010

Hof11 N. Hoffmann, Independent parameters for special instanton bundles on $\mathbb{P}^{2 n+1}$, J. Geom. Phys. 61 (2011), 2321-2330. http://dx.doi.org/10.1016/j.geomphys.2011.07.006

Hul80 K. Hulek, On the classification of stable rank-r vector bundles over the projective plane, in Vector bundles and differential equations (Proc. Conf., Nice, 1979), 113-144, Progr. Math., 7, Birkhäuser, Boston, Mass., 1980.

JV11 M. Jardim and M. Verbitsky, Trihyperkähler reduction and instanton bundles on $\mathbb{C P}^{3}$, Compositio Math., to appear, arXiv:1103.4431.

Kin94 A.D. King, Moduli of representations of finite-dimensional algebras, Quart. J. Math. Oxford Ser. (2) 45 (1994), 515-530.

MFK94 D. Mumford, J. Fogarty, and F. Kirwan, Geometric invariant theory, third edition, Ergebnisse der Mathematik und ihrer Grenzgebiete (2), vol. 34, Springer-Verlag, Berlin, 1994.

MT10 D. Markushevich and A.S. Tikhomirov, Rationality of instanton moduli arXiv:1012.4132v1 (2010).

OS83 Ch. Okonek and H. Spindler, Stabile reflexive Garben vom Rang 3 auf $\mathbb{P}^{3}$ mit kleinen Chernklassen, Math. Ann. 264 (1983), 91-118.

OS86 Ch. Okonek and H. Spindler, Mathematical instanton bundles on $\mathbb{P}^{2 n+1}$, J. reine angew. Math. 364 (1986), 35-50.

OSS80 Ch. Okonek, M. Schneider, and H. Spindler, Vector bundles on complex projective spaces, Progress in Mathematics, 3, Birkhäuser, Boston-Basel-Stuttgart, 1980.

OST99 Ch. Okonek, A. Schmitt, and A. Teleman, Master spaces for stable pairs, Topology 38 (1999), 117-139.

Ott96 G. Ottaviani, Real and complex 't Hooft instanton bundles over $\mathbb{P}^{2 n+1}(\mathbb{C})$, Rend. Semin. Mat. Fis. Milano 66 (1996), 169-199.

Rao97 A.P. Rao, Mathematical instantons with maximal order jumping lines, Pacific Journal of Math. 178 (1997), 331-344. 


\section{Costa, N. Hoffmann, R.M. Miró-Roig, A. Schmitt}

Sala84 S.M. Salamon, Quaternionic structures and twistor spaces, in Global Riemannian geometry (Durham, 1983), edited by T.J. Willmore and N.J. Hitchin, 65-74, Ellis Horwood Ser. Math. Appl., Horwood, Chichester, 1984.

Salt02 D.J. Saltman, Invariant fields of symplectic and orthogonal groups, J. Algebra 258 (2002), 507534. http://dx.doi.org/10.1016/S0021-8693(02)00640-3

Sch08 A.H.W. Schmitt, Geometric invariant theory and decorated principal bundles, Zurich Lectures in Advanced Mathematics, European Mathematical Society (EMS), Zürich, 2008. http://dx. doi.org/10.4171/065

Ser08 J.-P. Serre, Espaces fibrés algébriques, Seminaire Claude Chevalley, 1958, Documents Mathématiques, vol. 1, Soc. Math. France, 2008, 107-140.

Ski97 M. Skiti, Sur une famille de fibrés instantons, Math. Z. 225 (1997), 373-394.

ST90 H. Spindler and G. Trautmann, Special instanton bundles on $\mathbb{P}_{2 N+1}$, their geometry and their moduli, Math. Ann. 286 (1990), 559-592.

Tik12 A.S. Tikhomirov, Moduli of mathematical instanton vector bundles with odd $c_{2}$ on projective space, (Russian) Izv. Ross. Akad. Nauk Ser. Mat. 76 (2012), 143-224; translation in Izv. Math. 76 (2012), 991-1073. http://dx.doi.org/10.1070/IM2012v076n05ABEH002613

L. Costa costa@ub.edu

Facultat de Matemàtiques, Departament d'Algebra i Geometria, Gran Via de les Corts Catalanes 585, 08007 Barcelona, Spain

N. Hoffmann norbert.hoffmann@mic.ul.ie

Department of Mathematics and Computer Studies, Mary Immaculate College, South Circular Road, Limerick, Ireland

R.M. Miró-Roig miro@ub.edu

Facultat de Matemàtiques, Departament d'Algebra i Geometria, Gran Via de les Corts Catalanes 585, 08007 Barcelona, Spain

A. Schmitt alexander.schmitt@fu-berlin.de

Institut für Mathematik, Freie Universität Berlin, Arnimallee 3, 14195 Berlin, Germany 